\title{
The molecular basis of monopolin recruitment to the kinetochore
}

\author{
Rebecca Plowman $^{1}$ - Namit Singh ${ }^{2,3}$. Eelco C. Tromer ${ }^{4,5,6,7}$. Angel Payan ${ }^{8,9} \cdot$ Eris Duro $^{1} \cdot$ Christos Spanos $^{1}$. \\ Juri Rappsilber $^{1,10} \cdot$ Berend Snel $^{4} \cdot$ Geert J. P.L. Kops ${ }^{5,6} \cdot$ Kevin D. Corbett $^{8,9} \cdot$ Adele L. Marston $^{1}$
}

Received: 30 October 2018 / Revised: 8 March 2019 / Accepted: 19 March 2019/Published online: 30 April 2019

(C) The Author(s) 2019

\begin{abstract}
The monopolin complex is a multifunctional molecular crosslinker, which in S. pombe binds and organises mitotic kinetochores to prevent aberrant kinetochore-microtubule interactions. In the budding yeast $S$. cerevisiae, whose kinetochores bind a single microtubule, the monopolin complex crosslinks and mono-orients sister kinetochores in meiosis I, enabling the biorientation and segregation of homologs. Here, we show that both the monopolin complex subunit Csm1 and its binding site on the kinetochore protein Dsn1 are broadly distributed throughout eukaryotes, suggesting a conserved role in kinetochore organisation and function. We find that budding yeast Csm1 binds two conserved motifs in Dsn1, one (termed Box 1) representing the ancestral, widely conserved monopolin binding motif and a second (termed Box 2-3) with a likely role in enforcing specificity of sister kinetochore crosslinking. We find that Box 1 and Box 2-3 bind the same conserved hydrophobic cavity on Csm1, suggesting competition or handoff between these motifs. Using structure-based mutants, we also find that both Box 1 and Box 2-3 are critical for monopolin function in meiosis. We identify two conserved serine residues in Box 2-3 that are phosphorylated in meiosis and whose mutation to aspartate stabilises Csm1-Dsn1 binding, suggesting that regulated phosphorylation of these residues may play a role in sister kinetochore crosslinking specificity. Overall, our results reveal the monopolin complex as a broadly conserved kinetochore organiser in eukaryotes, which budding yeast have co-opted to mediate sister kinetochore crosslinking through the addition of a second, regulatable monopolin binding interface.
\end{abstract}

Keywords Monopolin $\cdot$ Kinetochore $\cdot$ RWD domain $\cdot$ Meiosis

Rebecca Plowman and Namit Singh contributed equally to this work.

This article is part of a Special Issue on Recent advances in meiosis from DNA replication to chromosome segregation edited by Valérie Borde and Francesca Cole, co-edited by Paula Cohen and Scott Keeney

Electronic supplementary material The online version of this article (https://doi.org/10.1007/s00412-019-00700-0) contains supplementary material, which is available to authorized users.

Kevin D. Corbett

kcorbett@ucsd.edu

$\triangle$ Adele L. Marston

adele.marston@ed.ac.uk

1 Wellcome Centre for Cell Biology, School of Biological Sciences, University of Edinburgh, Max Born Crescent, Edinburgh EH9 3BF, UK

2 Ludwig Institute for Cancer Research, San Diego Branch, La Jolla, CA 92093, USA

3 Present address: Synthorx Inc., 11099 North Torrey Pines Road, Suite 290, La Jolla, CA 92037, USA

4 Theoretical Biology and Bioinformatics, Biology, Science Faculty, Utrecht University, Utrecht, The Netherlands
5 Oncode Institute, Hubrecht Institute-KNAW (Royal Netherlands Academy of Arts and Sciences), Utrecht, The Netherlands

6 University Medical Centre Utrecht, Utrecht, The Netherlands

7 Department of Biochemistry, University of Cambridge, Cambridge, UK

8 Department of Cellular and Molecular Medicine, University of California, San Diego, La Jolla, CA 92093, USA

9 Department of Chemistry, University of California, San Diego, La Jolla, CA 92093, USA

10 Institute of Biotechnology, Technische Universität Berlin, Berlin, Germany 


\section{Introduction}

Meiosis generates haploid gametes from a diploid progenitor cell through two consecutive rounds of chromosome segregation that follow a single round of DNA replication (reviewed in (Duro and Marston 2015)). The first meiotic division (meiosis I) requires that the canonical chromosome segregation machinery be modified to direct the segregation of homologous chromosomes, rather than sister chromatids as in mitosis or meiosis II. Central to this process is the monoorientation of sister kinetochores, meaning that at metaphase I attachments are made to microtubules extending from the same spindle pole, rather than opposite poles, thereby ensuring the cosegregation of sister chromatids during anaphase I.

The mechanism of meiosis I sister kinetochore monoorientation is best understood in the budding yeast Saccharomyces cerevisiae. S. cerevisiae and its close relatives possess so-called "point centromeres," compact sequencedefined centromeres that bind a single centromeric nucleosome and assemble a minimal kinetochore (Meraldi et al. 2006; Westermann et al. 2007; Gordon et al. 2011). In S. cerevisiae meiosis I, sister kinetochores are fused through the action of the kinetochore-binding monopolin complex, and together bind a single microtubule (Winey et al. 2005; Corbett et al. 2010; Corbett and Harrison 2012; Sarangapani et al. 2014). The conserved core of the monopolin complex comprises two nucleolar proteins, Csm1 and Lrs4 (Rabitsch et al. 2003). These proteins form a distinctive V-shaped complex, with two Csm1 homodimers bridged at their coiled-coil $\mathrm{N}$-termini by a pair of Lrs4 subunits, thereby positioning two pairs of Csm1 globular-domain "heads" $\sim 10 \mathrm{~nm}$ apart at the apices of the V (Corbett et al. 2010). Each Csm1 globular domain has a conserved hydrophobic cavity implicated in binding the kinetochore protein Dsn1, leading to the proposal that monopolin could bridge Dsn 1 molecules from sister kinetochores to physically fuse the kinetochores (Corbett et al. 2010). Supporting this idea, kinetochore particles purified from cells in meiosis I bind microtubules more strongly than those from cells in mitosis or meiosis II, and this increased strength depends on the monopolin complex (Sarangapani et al. 2014). Further, addition of recombinant monopolin complex to kinetochores purified from mitotic cells increases their microtubule-attachment strength to match that of meiosis I kinetochores (Sarangapani et al. 2014).

A key unresolved question in monopolin function is how the complex specifically recognises and crosslinks sister kinetochores. This specificity is likely mediated by two additional monopolin complex subunits, the meiosis-specific protein Mam1 and a CK1 $\delta$ family kinase, Hrr25 (Toth et al. 2000; Rabitsch et al. 2003; Petronczki et al. 2006). Mam1, which is found only in point-centromere fungi, binds Csm1 and Hrr25 independently, through two flexibly linked domains, thereby acting as a molecular tether to recruit Hrr25 to the monopolin complex (Corbett and Harrison 2012; Ye et al. 2016). While CK1 $\delta$ family kinases are near-universal in eukaryotes, Hrr25 orthologs in point-centromere fungi possess a central domain that binds Mam1 and may uniquely regulate the protein's kinase activity when it is associated with the monopolin complex (Ye et al. 2016). While the relevant substrates of monopolin-associated Hrr25 have not been identified, the flexibility and length $(\sim 120 \AA)$ of the Mam1 tether would allow the kinase to access potential substrates within both monopolin and the kinetochore (Corbett and Harrison 2012; Ye et al. 2016). One candidate target is the kinetochore receptor for monopolin, Dsn1, which we previously showed is phosphorylated in vitro by Hrr25 (Ye et al. 2016). Hrr25's kinase activity is dispensable for kinetochore localisation of the monopolin complex in vivo (Petronczki et al. 2006) and for fusion of purified kinetochore particles in vitro (Sarangapani et al. 2014), but is required for sister kinetochore monoorientation in meiosis I (Petronczki et al. 2006). Together, these data suggest that kinetochore binding is functionally distinct from sister kinetochore crosslinking, and that Hrr25's kinase activity is specifically important for the latter.

Apart from its critical role at meiosis I kinetochores, the Csm1-Lrs4 monopolin subcomplex acts as a molecular crosslinker in at least three other functional contexts in S. cerevisiae, some of which are likely conserved throughout fungi. Csm1 and Lrs4 reside in the nucleolus for the majority of the cell cycle, and a subset of Csm1-Lrs4 is released from the nucleolus after meiotic prophase to function at meiotic kinetochores (Rabitsch et al. 2003; Clyne et al. 2003). The complex is also released from the nucleolus in mitotic anaphase, when it localises to kinetochores independently of Mam1 and Hrr25, and appears to suppress chromosome loss through an unknown mechanism (Brito et al. 2010). Within the nucleolus, Csm1 and Lrs4 are important for suppressing aberrant recombination within the highly repetitive ribosomal DNA (rDNA) repeats, and are also required for Sir2-mediated transcriptional silencing of rDNA (Huang et al. 2006; Mekhail et al. 2008). Csm1 binds the nucleolar protein Tof2 through the same conserved hydrophobic cavity implicated in Dsn1 binding, and also binds a SUMO peptidase, Ulp2, in a structurally equivalent manner to Mam1 (Liang et al. 2017). Finally, we have recently identified another Csm1-binding protein, Dse3, which binds Csm1 equivalently to Mam1 and Ulp2 (Singh and Corbett 2018). The biological role of the Dse3-Csm1 interaction is not known.

Outside point-centromere fungi, Csm1 and Lrs4 are also important in chromosome and kinetochore organisation and their molecular function is likely to be conserved. S. pombe Csm1 and Lrs4 (also called Pcs1 and Mde4) prevent aberrant chromosome-microtubule attachments in mitosis (Gregan et al. 2007; Choi et al. 2009) and have been proposed to do so through either physical crosslinking of microtubule binding sites within a single kinetochore, or alternatively through 
recruitment of chromosome-organising condensin complexes to centromeric chromatin (Tada et al. 2011). Condensindependent organisation of centromeres and rDNA is also thought to underlie the importance of Csm1-Lrs4 in the fungal pathogen Candida albicans (Burrack et al. 2013). While the monopolin complex is found throughout fungi, orthologs of Csm1 and Lrs4 have so far not been identified in other eukaryotes, questioning whether monopolin's kinetochoreorganising activities are broadly conserved.

While the architecture of the budding yeast monopolin complex and the structural basis for its interactions with numerous partners are known, direct molecular information about the monopolin-kinetochore interface is still lacking. A $\sim 40$-residue region within the disordered N-terminus of the core kinetochore protein, Dsn1, has been identified as the kinetochore receptor for the monopolin subunit Csm1 (Sarkar et al. 2013). This region, comprising residues $72-$ 110 of $S$. cerevisiae Dsn 1, is dispensable for vegetative growth but essential for sister kinetochore monoorientation in meiosis I (Sarkar et al. 2013). Sarkar et al. (2013) defined three conserved motifs in the Dsn1 72-110 region as Box 1, Box 2, and Box 3, and demonstrated their collective importance for Csm1 binding and monopolin function (Sarkar et al. 2013). Here, we combine comparative genomics of the kinetochore in eukaryotes and structural analysis of reconstituted Csm1-Dsn1 complexes with targeted mutagenesis, genetics, and imaging to dissect the molecular basis for monopolin recruitment and sister kinetochore monoorientation. We find that the Dsn1 Box 1 and Box 2-3 regions can each bind the conserved hydrophobic cavity on Csm1, and that these two interaction modes are mutually exclusive in a given Csm1-Dsn1 complex. We demonstrate that both interfaces are required for robust monopolin recruitment to kinetochores and for sister kinetochore monoorientation, and that simultaneous disruption of both interfaces leads to additive effects on meiosis. We show that both Csm1 and Dsn1 Box 1 are widely conserved in eukaryotes and provide evidence, using $S$. pombe proteins, that Box 1 is the ancestral kinetochore receptor for monopolin. The Dsn1 Box 2-3 region, meanwhile, is conserved only in point-centromere fungi and likely represents an adaptation to the complex's meiotic functions. Further, Dsn 1 Box 3 contains two conserved serine residues that are phosphorylated to modulate Dsn1-Csm1 binding, providing a potential molecular mechanism for sister kinetochore crosslinking specificity in meiosis I.

\section{Materials and methods}

\section{Proteome database}

We compiled a database of 109 proteomes based on sets that our labs used in previous studies. For the versions and sources of the selected proteomes, we therefore refer to two studies of van Hooff et al. (van Hooff et al. 2017a, b). Notable exceptions are the proteomes of Bombyx mori, Nasonia virtripennis and Agaricus bisporus, which we have downloaded on January 12, 2018, from the Ensembl genomes database (http://ensemblgenomes.org/). In addition, we received the proteome of the amoebozoa Physarum polycephalum from the lab of Pauline Schaap (see for contigs http://www. physarum-blast.ovgu.de/).

\section{Orthologs}

To create our set of orthologs we searched the 109 proteomes using our in-house established kinetochore HMM profiles of CCAN/Ctf19 complex and KMN network proteins (van Hooff et al. 2017a). In cases where HMM profile searches were incomplete or inconclusive we manually searched for orthologs using previously established procedures and criteria (van Hooff et al. 2017a). In addition, we performed phylogenetic profiling of 5 lineage-specific kinetochore proteins that were not included in our previous analyses (Csm1, Lrs4, Mam1, Nkp1 and Nkp2). We excluded Hrr25, since the resolution of kinase evolution and the accurate calling of Hrr25 orthologs requires further in-depth analysis. In addition, since most eukaryotes likely have an Hrr25 ortholog, we assumed that its phylogenetic profiles would not be informative in our analysis. See Table S1 and Supplementary Sequences for presence-absence profiles and sequence information of all orthologs reported in this study.

\section{Gene search and gene prediction}

To systematically search for genes that were absent in our previous analyses, we adopted 3 strategies: (1) we used our custom made HMM models of either orthologous groups or specific features such as domains and motifs, to search for a gene of interest in six-frame translated genome contigs, (2) we used an orthologous sequence of a closely related species to query whole genome shotgun sequences using tblastn, (3) we used an orthologous sequence of a closely related species to query six-frame translated genome contigs using phmmer. To assess sequence quality issues, we manually flagged incomplete proteins based on multiple sequence alignments of orthologous protein families. Proteins were deemed incomplete in cases where at least stretches of 15 amino acids were found missing. Common mistakes include incorrect gene fissions and fusions and wrongly omitted exons. Predicted or incomplete gene regions were extended with $<50,000 \mathrm{bp}$ and used to predict a gene by GENESCAN (Burge and Karlin 1997) and AUGUSTUS (Stanke et al. 2006), using various species-specific models. 


\section{Conserved feature extraction pipeline and co-evolutionary analysis}

The pipeline we used to uncover the Dsn1-N (Box 1) motif in a wide distribution of eukaryotes is based on a previously established workflow termed ConFeaX (Tromer et al. 2016). Orthologous sequences were masked using IUpred (Dosztányi et al. 2005) (disorder/order threshold $=0.4$ ) and MARCOIL (Delorenzi and Speed 2002) (coiled-coil threshold =90). ConFeaX starts with a probabilistic search for short conserved regions (6-100 aa) in masked orthologs using the MEME algorithm (option: any number of repeats) (Bailey et al. 2009). Significant motif hits are extended on both sides by five residues to compensate for the strict treatment of alignment information by the MEME algorithm and aligned using MAFFT-LINSI (Katoh and Standley 2013) to introduce gaps. The alignments were modelled using the HMMER packing (Eddy 2011) and sensitive profile HMM searches (using jackhmmer) were iterated (E-value $=1)$ until convergence. In some cases, we manually optimised the HMM profile searches using permissive bit scores and removed obvious false hits. Subsequently, for each of the conserved features, a phylogenetic profile was derived (present is ' 1 ' and absent is ' 0 '). For all possible pairs, we determined the correlation/ similarity using Pearson correlation coefficient (Wu et al. 2003). Pearson distances $(D=1-r)$ were used to map the phylogenetic profile similarity of kinetochore proteins in $2 \mathrm{D}$ using Barnes-Hut t-SNE (Maaten and Hinton 2008) (R-package 'Rtsne' [perplexity $=5$, dimensions $=2$ and theta $=0$ ], see Fig. 1). Sequence logos depicted throughout this study were obtained using weblogo2 (Crooks et al. 2004).

\section{Cloning and protein purification}

All protein coding sequences were amplified from genomic DNA and cloned into pET-based vectors, either without tags or encoding N-terminal TEV protease-cleavable $\mathrm{His}_{6}$ or $\mathrm{His}_{6}$ SUMO tags. Coexpression cassettes were generated by PCR and re-inserted into the same vectors. Point-mutations were generated by PCR. For expression, vectors were transformed into $E$. coli Rosetta2 (DE3) pLysS cells (EMD Millipore), and cultures were grown at $37{ }^{\circ} \mathrm{C}$ to an absorbance at $600 \mathrm{~nm}$ of 0.8 . The cultures were shifted to $20^{\circ} \mathrm{C}$ and protein expression was induced by the addition of $0.25 \mathrm{mM}$ IPTG, and cells were grown $\sim 16 \mathrm{~h}$ before harvesting by centrifugation.

For protein purification, cells were resuspended in protein buffer $(20 \mathrm{mM}$ Tris- $\mathrm{HCl} \mathrm{pH} 7.5,5 \%$ glycerol, $2 \mathrm{mM} \beta$ mercaptoethanol) plus $300 \mathrm{mM} \mathrm{NaCl}$ and $10 \mathrm{mM}$ imidazole, lysed by sonication, and centrifuged $30 \mathrm{~min}$ at $17,000 \mathrm{rpm}$ to remove cell debris. The supernatant was loaded onto a $5-\mathrm{mL}$ Histrap HP column (GE Life Sciences), washed with protein buffer plus $300 \mathrm{mM} \mathrm{NaCl} / 20 \mathrm{mM}$ imidazole, then with protein buffer plus $100 \mathrm{mM} \mathrm{NaCl} / 20 \mathrm{mM}$ Imidazole. Protein was eluted with protein buffer plus $100 \mathrm{mM} \mathrm{NaCl} / 250 \mathrm{mM}$ imidazole. Protein was then loaded onto a $5 \mathrm{~mL}$ Hitrap Q HP column (GE Life Sciences), washed with protein buffer plus $100 \mathrm{mM} \mathrm{NaCl}$, then eluted with a gradient to $600 \mathrm{mM} \mathrm{NaCl}$. Peak fractions were pooled, and TEV protease (Tropea et al. 2009) was added to cleave $\mathrm{His}_{6}$ or $\mathrm{His}_{6}-\mathrm{SUMO}$ tags, and the mixture was incubated $16 \mathrm{~h}$ at $4{ }^{\circ} \mathrm{C}$ (for $C g \mathrm{Csm} 1^{69-181}: S \mathrm{C}$ $\mathrm{His}_{6}-\mathrm{Dsn} 1^{71-110}$ and $C g \mathrm{His}_{6}-\mathrm{Csm}^{69-181}: \mathrm{SCDsn}^{71-110}$, tag cleavage was not performed; eluted fractions were instead concentrated and passed directly over a Superdex 200 column). After tag cleavage, the mixture was passed over Histrap HP and the flow-through collected, concentrated by ultrafiltration (Amicon Ultra, EMD Millipore), then passed over a HiLoad Superdex 200 size exclusion column (GE Life Sciences) in protein buffer plus $300 \mathrm{mM} \mathrm{NaCl}$ (with $1 \mathrm{mM}$ dithiothreitol substituting for $\beta$-mercaptoethanol) for final purification. Protein was exchanged into buffer containing $20 \mathrm{mM}$ Tris- $\mathrm{HCl} \mathrm{pH} \mathrm{7.5,} 100 \mathrm{mM} \mathrm{NaCl}$, and $1 \mathrm{mM}$ DTT, concentrated to $\sim 10 \mathrm{mg} / \mathrm{mL}$, and stored at $4{ }^{\circ} \mathrm{C}$ for crystallisation.

\section{Crystallisation and structure determination}

CgCsm $1^{69-181}:$ CgMam $1^{162-216}$ For crystallisation of the $C g C s m 1^{69-181}: C g M a m 11^{162-216}$ complex, purified protein at $10 \mathrm{mg} / \mathrm{mL}$ was mixed $1: 1$ with well solution containing $0.1 \mathrm{M}$ MES pH 6.5, 0.6 M NaCl, and 20\% PEG 4000. Crystals were cryoprotected with the addition of $20 \%$ PEG 400 and flash-frozen in liquid nitrogen. Diffraction data were collected to $3.03 \AA$ resolution at the Advanced Photon Source, NE-CAT beamline 24ID-E (support statement below) and

Fig. 1 Identification of Csm1 and a conserved N-terminal Dsn 1 motif in a wide range of eukaryotes. a Speculative model for intra-kinetochore crosslinking by monopolin in mitosis, based on prior observations that the budding yeast monopolin complex subunit Csm1 interacts with the kinetochore through a disordered region in the Mis 12 complex subunit Dsn1. Using our previously established workflow ConFeaX (Tromer et al. 2016), we uncovered a short motif (Dsn1-N) that is conserved in a wide range of eukaryotic Dsn1 orthologs (Fig. S1). b Presence-absence profiles of the KMN network (including Knl1/Zwint-1, Mis12 complex, and the Ndc80 complex), CCAN/Ctf19 complex, plus Csm1 and Dsn1-N in 109 eukaryotic proteomes. White squares indicate absence and coloured squares presence of the proteins in a particular species (colours correspond to complexes in panel A). The tree to the right depicts the various eukaryotic supergroups. Encephalitozoon and Oomycetes are highlighted to indicate that these species' Dsn1 proteins appear to possess two Dsn1-N motifs (Fig. S1b, c). c $t$-SNE projection and 2 dimensional representation of phylogenetic profile similarity (Pearson distance $[D=$ $1--r]$ ) of kinetochore proteins depicted in panel $b$. The table in the lower left corner summarises the frequencies of Csm1, Dsn1 and Dsn1-N in 109 eukaryotic species (panel b). While the presence-absence profiles of Dsn 1 and Csm1 are not similar (Pearson correlation coefficient, $r=0.339$ ), the presence-absence profiles of Csm1 and Dsn1-N are highly similar $(r=$ 0.799). In species with both Csm1 and Dsn1, only 6 do not have a Dsn1$\mathrm{N}$ motif (6 of 55), while in species with Dsn1 that lack Csm1, none have the Dsn1-N motif (0 of 30) 

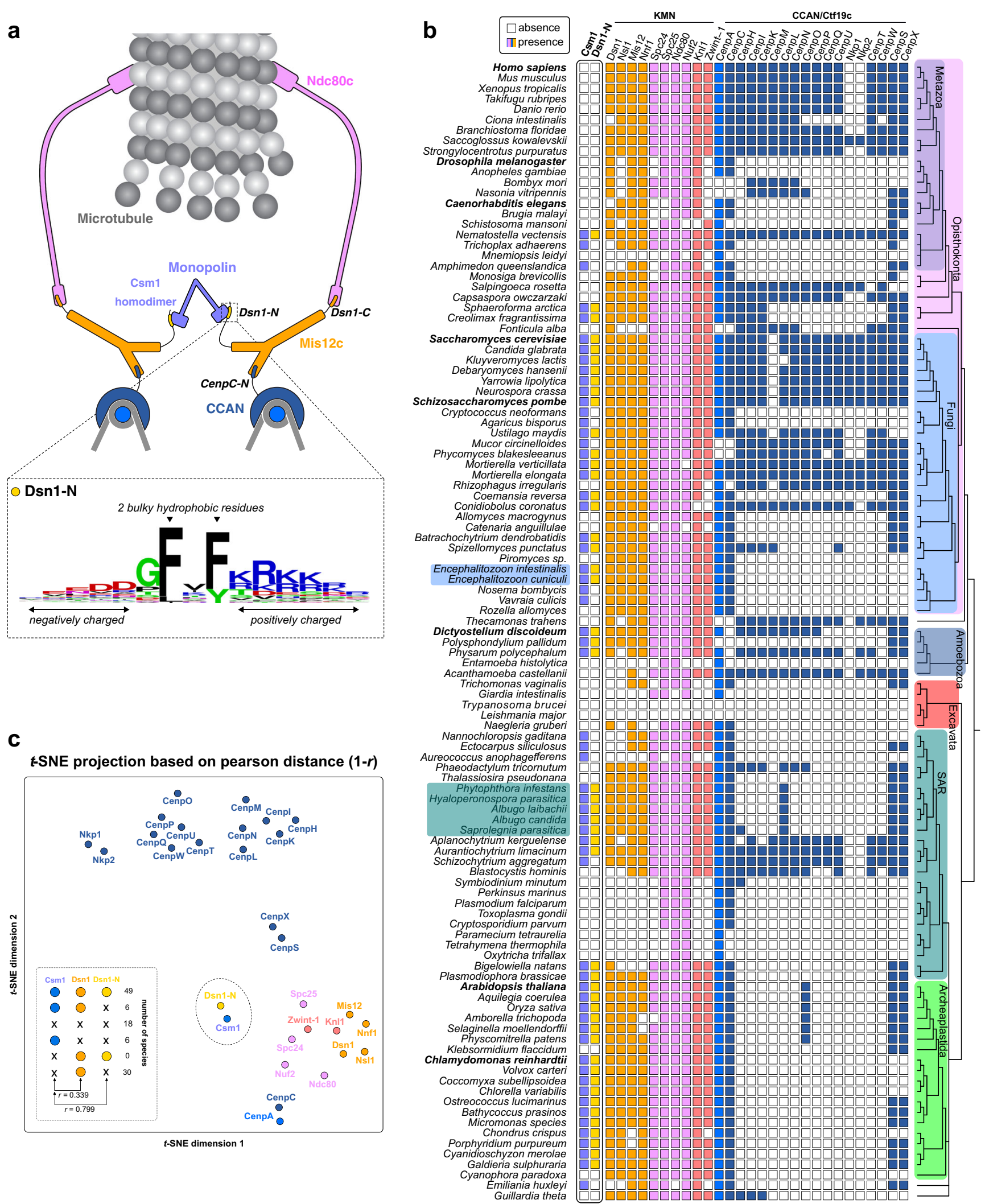

$t$ SNE projection based on pearson distance (1-r)

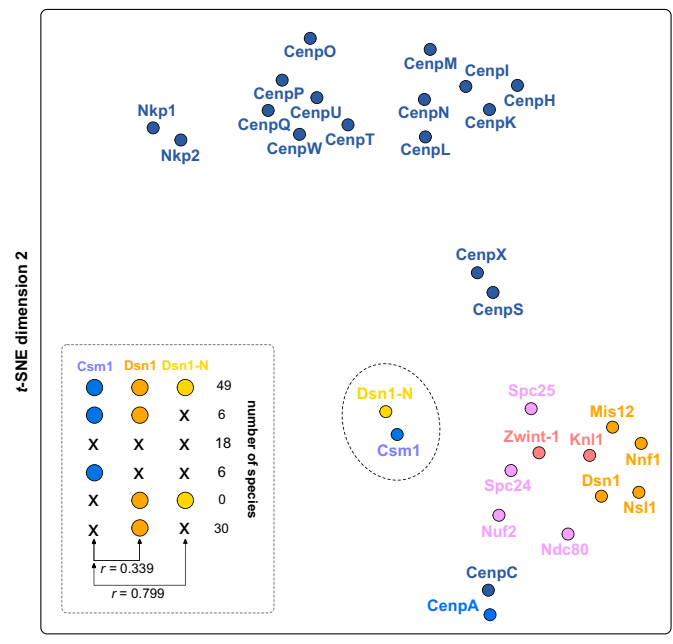

t-SNE dimension 1

indexed/reduced with the RAPD automated data-processing pipeline (https://github.com/RAPD/RAPD), which uses XDS (Kabsch 2010) for indexing and integration, and the CCP4 programmes AIMLESS (Evans and Murshudov 2013) and TRUNCATE (Winn et al. 2011) for scaling and structurefactor calculation. The structure was determined by molecular 
replacement in PHASER (McCoy et al. 2007) using the structure of $S$. cerevisiae Csm1 (PDB ID 3N4R) (Corbett et al. 2010) as a search model. The model, including all $C g$ Mam 1 residues, was manually built in COOT (Emsley et al. 2010) and refined in phenix.refine (Afonine et al. 2012) using positional, individual B-factor, and TLS refinement (Table S5).

CgCsm1 $1^{69-181}:$ CgDsn $1^{14-72}$ For crystallisation of the $C g C s m 1^{69-181}: C g D s n 1^{14-72}$ complex, purified protein at $10 \mathrm{mg} / \mathrm{mL}$ was mixed $1: 1$ with well solution containing $0.45 \mathrm{M}$ Ammonium sulphate, 5\% PEG 3350, and $0.1 \mathrm{M}$ Bis-Tris, pH 5.5 in hanging-drop format at $20{ }^{\circ} \mathrm{C}$. Crystals were cryoprotected with the addition of $25 \%$ glycerol and flash-frozen in liquid nitrogen. Diffraction data were collected to $2.27 \AA$ resolution at the Advanced Photon Source, NE-CAT beamline 24ID-E and indexed/reduced with the RAPD automated data-processing pipeline. The structure was determined by molecular replacement, manually rebuilt and refined as above.

$\mathrm{Cg} \mathrm{His}{ }_{6}-\mathrm{Csm}^{69-181}: \mathrm{SCDsn1}^{71-110}$ For crystallisation of the $\mathrm{Cg}$ $\mathrm{His}_{6}-\mathrm{Csm}^{69-181}: \mathrm{ScDsn1}^{71-110}$ complex, purified protein at $10 \mathrm{mg} / \mathrm{mL}$ was mixed $1: 1$ with well solution containing $0.2 \mathrm{M}$ $\mathrm{MgCl}_{2}, 0.1 \mathrm{M}$ Tris-HCl pH 8.5, and 25\% PEG 3350. Crystals were cryoprotected with the addition of $20 \%$ PEG 400 and flashfrozen in liquid nitrogen. Diffraction data were collected to $2.5 \AA$ resolution at the Stanford Synchrotron Radiation Laboratory, beamline 14-1 (support statement below). Data were indexed, reduced, and scaled with HKL2000 (Otwinowski and Minor 1997) and converted to structure factors using TRUNCATE (Winn et al. 2011). The structure was determined by molecular replacement, manually rebuilt and refined as above.

$C g C s m 1^{69-181}: C g D s n 1^{43-67}$ DD For crystallisation of the $C g C s m 1^{69-181}: C g D s n 1^{43-67} \mathrm{DD}$ complex (serines 66 and 67 mutated to aspartate), purified protein at $10 \mathrm{mg} / \mathrm{mL}$ was mixed 1:1 with well solution containing $0.1 \mathrm{M}$ Sodium acetate $\mathrm{pH} 4.5$ and $3 \mathrm{M} \mathrm{NaCl}$. Crystals were cryoprotected with the addition of 2.5 M Sodium Malonate $\mathrm{pH} 4.5$ and flash-frozen in liquid nitrogen. Diffraction data were collected at the Advanced Photon Source, NE-CAT beamline 24ID-E and indexed/reduced with the RAPD automated data-processing pipeline. The structure was determined by molecular replacement, manually rebuilt and refined as above.

All macromolecular structure figures were generated with PyMOL version 2.2 (Schrödinger, LLC), and surface charge for Fig. $4 \mathrm{f}$ was calculated using the APBS (Jurrus et al. 2018) plugin for PyMOL.

\section{Synchrotron support statements}

Advanced photon source This work is based upon research conducted at the Northeastern Collaborative Access Team beamlines, which are funded by the National Institute of General Medical Sciences from the National Institutes of Health (P30 GM124165). The Eiger 16 M detector on 24ID-E beam line is funded by a NIH-ORIP HEI grant (S10OD021527). This research used resources of the Advanced Photon Source, a U.S. Department of Energy (DOE) Office of Science User Facility operated for the DOE Office of Science by Argonne National Laboratory under Contract No. DE-AC02-06CH11357.

Stanford synchrotron radiation Lightsource Use of the Stanford Synchrotron Radiation Lightsource, SLAC National Accelerator Laboratory, is supported by the U.S. Department of Energy, Office of Science, Office of Basic Energy Sciences under Contract No. DE-AC02-76SF00515. The SSRL Structural Molecular Biology Program is supported by the DOE Office of Biological and Environmental Research, and by the National Institutes of Health, National Institute of General Medical Sciences (including P41GM103393). The contents of this publication are solely the responsibility of the authors and do not necessarily represent the official views of NIGMS or NIH.

\section{Protein-protein interaction assays}

For in vitro translation and $\mathrm{Ni}^{2+}$ pulldown assays, S. pombe Mis13 (Dsn1) residues 1-100 and S. cerevisiae Dsn1 residues 71-110 (and point mutants thereof) were cloned with an Nterminal maltose binding protein tag (no His $_{6}$-tag) into a pETbased vector with a Kozak sequence immediately upstream of the coding sequence. These vectors were used as a template for in vitro transcription/translation using a TNT T7 coupled transcription/translation kit (Promega) in the presence of ${ }^{35} \mathrm{~S}$ labelled methionine to generate prey proteins for pulldowns. Ten microliters of transcribed protein mix was incubated with $10 \mu \mathrm{g} \mathrm{His}_{6}$-tagged bait protein (S. pombe $\mathrm{Csm}^{125-261}$ or S. cerevisiae $\mathrm{Csm}^{69-190}$ ) in $50 \mu \mathrm{l}$ buffer (20 mM HEPES, $\mathrm{pH} 7.5,150 \mathrm{mM} \mathrm{NaCl}, 20 \mathrm{mM}$ imidazole, $5 \%$ glycerol, $1 \mathrm{mM}$ dithiothreitol (DTT), $0.1 \%$ NP-40) for 90 min at $4{ }^{\circ} \mathrm{C}$, then $15 \mu \mathrm{l}$ Ni-NTA beads were added, and the mixture was incubated a further $45 \mathrm{~min}$. Beads were washed three times with $0.5 \mathrm{~mL}$ buffer, then eluted with $25 \mu \mathrm{L}$ elution buffer $(2 \times$ SDS-PAGE loading dye plus $400 \mathrm{mM}$ imidazole) and boiled. Samples were run on SDS-PAGE, dried, and scanned with a phosphorimager.

For fluorescence polarisation peptide-binding assays, purified S. pombe Csm1 $1^{125-261}$ (wild type or I241D mutant, equivalent to $S c$ Csm1 L161D) at $20 \mathrm{nM}-250 \mu \mathrm{M}$ was incubated with 20 nM $S p$ Mis13 5-17 peptide (fluorescein isothiocyanate-labelled at its N-terminus) in a buffer containing $20 \mathrm{mM}$ Tris $7.5,300 \mathrm{mM} \mathrm{NaCl}, 10 \%$ glycerol, $0.01 \% \mathrm{NP}-$ 40 , and $1 \mathrm{mM}$ DTT ( $50 \mu \mathrm{L}$ reactions, measured in triplicate). Binding data were fit to a single-site binding model with Prism version 7 (Graphpad Software). 


\section{Isothermal titration calorimetry}

Isothermal titration calorimetry was performed on a Microcal ITC 200 (Malvern Panalytical) in protein buffer plus $300 \mathrm{mM}$ $\mathrm{NaCl}$ and $1 \mathrm{mM}$ dithiothreitol. $\mathrm{His}_{6}$-MBP-fused Dsn1 fragments at $1-1.5 \mathrm{mM}$ were injected into a sample cell containing untagged $\mathrm{Csm} 1$ at $100-200 \mu \mathrm{M}$.

\section{Yeast strains and plasmids}

Yeast strains used in this study were derivatives of SK1 with the exception of those for chromosome loss assays. All strains are given in Table S2. The CEN5-GFP marker consists of two components: (1) an array of tet operator sequences inserted at the chromosome $\mathrm{V}$ centromere, and (2) a Tet repressor protein fused to GFP, which binds to and specifically marks these operator sites and were previously described in (Toth et al. 2000). MAM1-9MYC was also described in (Toth et al. 2000) and MAM1-yeGFP was described in (Matos et al. 2008). PDS1-tdTomato and pCLB2-CDC20 were described in (Lee and Amon 2003) and (Matos et al. 2008), respectively. MTW1-tdTomato was generated in SK1 as described in (Fernius et al. 2013). $p C L B 2-C D C 20$ were described in (Lee and Amon 2003) and (Matos et al. 2008), respectively. $p G A L$ NDT80 pGPD1-GAL4(848)-ER for prophase I block release was described in (Benjamin et al. 2003). Chromosome III fragment (CFIII) for chromosome loss assays carrying HIS3 and Sup 11 was described in (Hieter et al. 1985). Point mutations in DSN1-6His-3FLAG were generated in plasmid pSB1590 (Akiyoshi et al. 2009) using the Quick Change IIXL kit (Agilent Technologies) and integrated into the DSN1 endogenous locus by PCR-mediated transformation. Plasmids generated in this study are given in Table S3.

\section{Yeast growth conditions}

Sporulation was induced as described by (Vincenten et al. 2015). Briefly, diploid yeast were grown overnight on YPG agar (1\% yeast extract, $2 \%$ Bacto peptone, $2.5 \%$ glycerol, and $2 \%$ agar), transferred to YPD4\% agar (1\% yeast extract, $2 \%$ Bacto peptone, $4 \%$ glucose, and $2 \%$ agar) and incubated for $24 \mathrm{~h}$ before inoculating into YEPD liquid medium (1\% yeast extract, $2 \%$ Bacto peptone, and $2 \%$ glucose) and incubating with shaking for $24 \mathrm{~h}$. Cells were transferred to BYTA (1\% yeast extract, $2 \%$ Bacto tryptone, $1 \%$ potassium acetate, $50 \mathrm{mM}$ potassium phthalate) at an $\mathrm{OD}_{600}=0.2-0.3$ or YPA ( $1 \%$ yeast extract, $2 \%$ tryptone peptone, $1 \%$ potassium acetate) and incubated for a further $\sim 16 \mathrm{~h}$. Cells were washed once with sterile distilled water and re-suspended in SPO medium $(0.3 \%$ potassium acetate, $\mathrm{pH} 7)$ at an $\mathrm{OD}_{600}=1.8-1.9$; $t=0$. Cells were incubated at $30^{\circ} \mathrm{C}$ for the duration.

\section{Benomyl sensitivity assay}

Haploid cells were grown at room temperature for $\sim 16 \mathrm{~h}$ in YEPD with shaking. Cultures were then diluted to an $\mathrm{OD}_{600}=0.1$ in water before making serial 1 in 10 dilutions. Dilutions were plated on either YPD agar or YPD containing $12 \%$ benomyl and incubated at $25^{\circ} \mathrm{C}$ for 3 days.

\section{Chromosome loss assay}

Assay measures the loss of chromosome III fragment (CFIII) carrying HIS3 and Sup11 described in (Hieter et al. 1985). Loss of Sup11, via loss of CFIII, stops suppression of ade21 mutation causing a colony colour change from white to red (Koshland and Hieter 1987). Cells were grown in minimal media lacking histidine for $\sim 16 \mathrm{~h}$ at room temperature. Cells were washed in YEPD liquid media without the addition of adenine. Cells were diluted to estimated 120 cells per plate and plated on YPD agar without the addition of adenine. After incubation at $25{ }^{\circ} \mathrm{C}$ for 5 days, the fraction of half-sectored colonies was scored. The number of half red colonies was divided by the total number of colonies to calculate the CFIII loss rate per cell division. Any completely red colonies were excluded as CFIII must have been lost prior to plating.

\section{Chromatin immunoprecipitation qPCR}

Cells carrying $p C L B 2-C D C 20$ (Lee and Amon 2003) were induced to sporulate. After $6 \mathrm{~h}$ in SPO, cells were fixed in $1 \%$ formaldehyde for $1 \mathrm{~h}$, washed twice with TBS (20 mM Tris- $\mathrm{HCl} \mathrm{pH} 7.5,150 \mathrm{mM} \mathrm{NaCl}$ ) and once with $1 \times \mathrm{FA}$ lysis buffer (50 mM HEPES-KOH at pH 7.5, $150 \mathrm{mM} \mathrm{NaCl}, 1 \mathrm{mM}$ EDTA, $1 \% \quad v / v$ Triton X-100, $0.1 \% w / v$ Sodium Deoxycholate) containing $0.1 \% \mathrm{w} / v$ SDS before resuspending in $1 \times$ FA lysis buffer $/ 0.1 \%$ SDS. Cells were lysed in a Fastprep Bio-pulveriser FP120 with silica beads (Biospec Products). Samples were sonicated to fragment chromosomal DNA using a BioRupter (Diagenode). Aliquots of the resultant chromatin solution were incubated with either anti-Myc (9E10, Biolegend) or anti-FLAG (M2, Sigma) antibodies and Protein G Dynabeads (Life Technologies) overnight at $4{ }^{\circ} \mathrm{C}$. Following sequential washes with CWB1 (FA lysis buffer/ $0.1 \%$ SDS/ $275 \mathrm{mM} \mathrm{NaCl}$ ), CWB2 (FA lysis buffer/0.1\% SDS/ $500 \mathrm{mM} \mathrm{NaCl}$ ), CWB3 (10 mM Tris-HCl, pH 8, $0.25 \mathrm{M} \mathrm{LiCl}, 1 \mathrm{mM}$ EDTA, $0.5 \% \mathrm{NP}-40,0.5 \% \mathrm{Na}$ Deoxycholate) and CWB4 (TE: $10 \mathrm{mM}$ Tris-HCl, $\mathrm{pH} 8$, 1 mM EDTA), immunoprecipitates and 1/100 input chromatin were recovered by boiling $(10 \mathrm{~min})$ with a $10 \%$ slurry of Chelex-100 resin before adding proteinase $\mathrm{K}(0.125 \mathrm{mg})$ and incubating at $55{ }^{\circ} \mathrm{C}$ for $30 \mathrm{~min}$, then boiled for a further $10 \mathrm{~min}$. Samples were centrifuged and supernatant taken for qPCR. qPCR was performed on a on a Roche Lightcycler with LUNA universal qPCR Master Mix (New England Biolabs). 
Primers used for qPCR are given in Table S4. To calculate ChIP enrichment/input, $\Delta \mathrm{CT}$ was calculated according to: $\Delta \mathrm{CT}=\left(\mathrm{CT}_{(\mathrm{ChIP})}-\left[\mathrm{CT}_{\text {(Input) }}-\log \mathrm{E}\right.\right.$ (input dilution factor) $\left.]\right)$ where $\mathrm{E}$ represents the specific primer efficiency value. Enrichment/input value was obtained from the following formula: $\mathrm{E}^{\wedge-\Delta \mathrm{CT}}$. $\mathrm{qPCR}$ was performed in triplicate from three or more independent cultures. Error bars represent standard error. Figures show the mean values for each strain, averaged over all individual experiments and biological replicates. Wild type and no tag controls were included for reference in all individual experiments and replicates. The number of replicates for each strain is indicated in the figure legends.

\section{Western blotting}

Samples for immunoblot analysis were fixed in 5\% TCA and cell pellets were washed once with acetone. Cells were lysed in $50 \mathrm{mM}$ Tris (pH 7.5), $1 \mathrm{mM}$ EDTA, and $50 \mathrm{mM}$ DTT containing protease inhibitors with glass beads, boiled in $1 \times$ sample buffer and visualised by detection of chemiluminesence on autoradiograms. Mouse Anti-FLAG M2 antibodies (Sigma) and mouse Anti-cMYC (9E10, Biolegend) were used at 1:1000 dilution, and rabbit anti-PGK1 (Marston lab stock) was used at $1: 10,000$ dilution.

\section{Spore viability}

Haploid yeast strains with the relevant genotypes were mated and single diploid colonies were incubated on SPO agar. A minimum of two diploid isolates were chosen for spore dissection. The total number of tetrads dissected for each strain is indicated in the figure legend. Spores were allowed to grow for 2 days on YPDA at $30{ }^{\circ} \mathrm{C}$ before scoring the number of viable colonies per tetrad.

\section{Fixed cell imaging}

Cells carrying $p C L B 2-C D C 20$ (Lee and Amon 2003) were induced to sporulate. After $6 \mathrm{~h}$ in SPO, cells were fixed in $3.7 \%$ formaldehyde for $10 \mathrm{~min}$, washed in $80 \%$ ethanol and suspended in DAPI $1 \mu \mathrm{g} / \mathrm{mL}$. Cells were counted as containing 1 or 2 GFP foci. Each strain was analysed in at least three independent biological repeats and the average is shown with standard error bars.

\section{Live cell imaging}

Cells were induced to sporulate as above. Cells were incubated $2 \mathrm{~h}$ in SPO medium in flasks for analysis of chromosome segregation. Alternatively, for Mam1 localisation, cells were incubated for $6 \mathrm{~h}$ in SPO before addition of $1 \mu \mathrm{M} \beta$-estradiol and incubated for a further $15 \mathrm{~min}$ to release cells from prophase I arrest. Cells were immobilised on Concanavalin A- coated cover slips in ibidi 4-well or 8-well dishes, fresh sporulation media was added to the dish and imaging commenced. Imaging was performed on a Zeiss Axio Observer Z1 (Zeiss UK, Cambridge) equipped with a Hamamatsu Flash 4 sCMOS camera, Prior motorised stage and Zen 2.3 acquisition software. Images were processed in Image J and $8 \mathrm{Z}$-stacks were projected to maximum intensity. Representative movies were generated using imaris, cells were projected to $2 \mathrm{D}$ using max intensities over the projection line (MIP) and contrast was adjusted to highlight florescent markers.

\section{Mass spectrometry}

Cells carrying $p C L B 2-C D C 20$ (Lee and Amon 2003) were induced to sporulate. After $6 \mathrm{~h}$ in SPO, cells were frozen. Kinetochores were isolated as described in (Akiyoshi et al. 2009) with some modifications. Extract was prepared by breaking yeast cells with a Retsch ball mill $(5 \times 3 \mathrm{~min}$ at $30 \mathrm{~Hz}$ for meiotic cells, with $5 \mathrm{~min}$ in liquid nitrogen in between) followed by ultracentrifugation $\left(24,000 \mathrm{rpm}\right.$ for $90 \mathrm{~min}$ at $\left.4{ }^{\circ} \mathrm{C}\right)$. Beads conjugated with anti-Flag antibodies were incubated with extract for $2.5 \mathrm{~h}$ with constant rotation, followed by three washes with buffer BH/0.15 (25 mM HEPES, $2 \mathrm{mM} \mathrm{MgCl} 2,0.1 \mathrm{mM}$ EDTA, $0.5 \mathrm{mM}$ EGTA pH 8.0, $0.1 \%$ NP- $40,150 \mathrm{mM} \mathrm{KCl}, 15 \%$ glycerol) containing protease inhibitors (at $10 \mu \mathrm{g} / \mathrm{mL}$ final concentration for each of chymostatin, leupeptin, antipain, pepstatin A, E-64, aprotinin; $2 \mathrm{mM}$ final AEBSF-Pefablock, $1 \mathrm{mM}$ NEM, $0.2 \mu \mathrm{M}$ microcystin and cOmplete EDTA-free Protease Inhibitor Cocktail (Sigma-Aldrich)) phosphatase inhibitors $(0.4 \mathrm{mM} \mathrm{Na}$ orthovanadate, $0.2 \mu \mathrm{M}$ microcystin, $4 \mathrm{mM} \beta$-glycerophosphate, $2 \mathrm{mM}$ Na pyrophosphate, $10 \mathrm{mM} \mathrm{NaF}$ ) and $2 \mathrm{mM}$ dithiothreitol (DTT). Beads were further washed twice with $\mathrm{BH} / 0.15$ with protease inhibitors. Beads were heated to $70{ }^{\circ} \mathrm{C}$ for $10 \mathrm{~min}$ in $50 \mathrm{mM}$ Tris pH 8 with $5 \%$ SDS, to elute the proteins.

Protein samples were run on SDS-PAGE (NuPAGE Novex 4-12\% Bis-Tris gel, Life Technologies, UK), in NuPAGE buffer (MES) and visualised using InstantBlue ${ }^{\mathrm{TM}}$ stain (Sigma-Aldrich, UK). The stained gel bands were excised and de-stained with $50 \mathrm{mM}$ ammonium bicarbonate (SigmaAldrich, UK) and $100 \%(v / v)$ acetonitrile (Sigma-Aldrich, UK) and in gel digestion was modified from (Shevchenko et al. 1996) to use AspN. In brief, proteins were reduced in $10 \mathrm{mM}$ dithiothreitol (Sigma-Aldrich, UK) for $30 \mathrm{~min}$ at $37{ }^{\circ} \mathrm{C}$ and alkylated in $55 \mathrm{mM}$ iodoacetamide (SigmaAldrich, UK) for $20 \mathrm{~min}$ at ambient temperature in the dark. They were then digested overnight at $37{ }^{\circ} \mathrm{C}$ with $13 \mathrm{ng} / \mu \mathrm{L}$ AspN (Promega, UK).

Phosphopeptides were enriched using a titanium dioxide $\left(\mathrm{TiO}_{2}\right)$ spin tips kit (High-Select ${ }^{\mathrm{TM}} \mathrm{TiO} 2$ Phosphopeptide Enrichment Kit, ThermoFisher Scientific). The sample was dried in vacuum centrifuge for storage. The flow through sample was loaded onto StageTip as described by Rappsilber et al. (2003), peptides were eluted in $40 \mu \mathrm{L}$ of 
$80 \%$ acetonitrile in $0.1 \%$ TFA and concentrated down to $1 \mu \mathrm{L}$ using a vacuum centrifuge (Concentrator 5301, Eppendorf, UK). Samples were prepared for LC-MS/MS analysis by diluting them to $6 \mu \mathrm{L}$ with $0.1 \%$ TFA. LC-MS-analyses were performed on an Orbitrap Fusion ${ }^{\mathrm{TM}}$ Lumos $^{\mathrm{TM}}$ Tribrid $^{\mathrm{TM}}$ Mass Spectrometer (Thermo Fisher Scientific, UK) coupled on-line to an Ultimate 3000 RSLCnano System (Dionex, Thermo Fisher Scientific, UK). Peptides were separated on a $75 \times$ $50 \mathrm{~cm}$ EASY-Spray column (2 $\mathrm{m}$ particle size, $100 \AA$, Thermo Fisher Scientific) assembled in an EASY-Spray source (Thermo Fisher Scientific, UK), operated at a constant temperature of $50{ }^{\circ} \mathrm{C}$. Peptides from the phospho-enriched samples were resuspended in $40 \mu \mathrm{L}$ of $0.1 \%$ TFA, vortexed and sonicated for $5 \mathrm{~min}$ and then concentrated down to $6 \mu \mathrm{L}$ with vacuum centrifugation, before they were injected on the mass spectrometer. For both sets of samples the same gradient and method were applied. Briefly, mobile phase A consisted of $0.1 \%$ formic acid in deionised water while mobile phase $\mathrm{B}$ consisted of $80 \%$ acetonitrile and $0.1 \%$ formic acid. Peptides were loaded onto the column at a flow rate of $0.3 \mu \mathrm{L} / \mathrm{min}$ and eluted at a flow rate of $0.25 \mu \mathrm{L} / \mathrm{min}$ according to the following gradient: 2 to $40 \%$ buffer B in $120 \mathrm{~min}$, then to $95 \%$ in $11 \mathrm{~min}$ (total run time of $160 \mathrm{~min}$ ). Survey scans were performed at 120,000 resolution (scan range $350-1500 \mathrm{~m} / \mathrm{z}$ ) with an ion target of 4.0e5. MS2 was performed in the ion trap at rapid scan mode with ion target of 2.0E4 and HCD fragmentation with normalised collision energy of 27 (Olsen et al. 2007). The isolation window in the quadrupole was set at 1.4 Thomson. Only ions with charge between 2 and 7 were selected for MS2.

The MaxQuant software platform (Cox and Mann 2008) version 1.6.1.0 was used to process raw files and search was conducted against the Saccharomyces cerevisiae (strain SK1) complete/reference proteome set of Saccharomyces Genome Database (released in December, 2016), using the Andromeda search engine (Cox et al. 2011). The first search peptide tolerance was set to $20 \mathrm{ppm}$ while the main search peptide tolerance was set to $4.5 \mathrm{pm}$. Isotope mass tolerance was $2 \mathrm{ppm}$ and maximum charge to 7 . AspN was chosen as a protease, allowing two missed cleavages. Carbamidomethylation of cysteine was set as fixed modification. Oxidation of methionine and acetylation of the N-terminal as well as phosphorylation of serine, threonine and tyrosine were set as variable modifications.

\section{Results}

\section{The monopolin complex subunit Csm1 is an ancient kinetochore component}

We previously reported extensive phylogenetic and evolutionary analysis of eukaryotic kinetochore subunits, but monopolin complex subunits were not included (van Hooff et al. 2017a). Reasoning that a role for monopolin in preventing merotelic kinetochore-microtubule attachments, as reported in $S$. pombe (Gregan et al. 2007; Rumpf et al. 2010; Tada et al. 2011), may be a more widely conserved function of the complex, we performed phylogenetic analysis of the Csm1 and Lrs4 monopolin subunits. We identified Csm1 orthologs in a wide variety of eukaryotic lineages outside fungi, including Archeaplastida (e.g. Arabidopsis thaliana Titan-9 and in Chlamydomonas reinhardtii) and Amoebozoa (e.g. Dictyostelium discoideum Cenp-68) (Fig. 1a, b). Based on this distribution, we conclude that Csm1 was likely a kinetochore subunit in the Last Eukaryotic Common Ancestor (LECA), and has since been lost from various eukaryotic lineages including in most metazoans. In contrast to Csm1, we could not detect orthologs of the Csm1 binding partner Lrs4 outside fungi. Since Lrs4 is predicted to be mostly unstructured (Corbett et al. 2010), its sequence likely diverges more quickly than Csm1, making any Lrs4 orthologs difficult to identify.

We next reasoned that species with Csm1 orthologs should also possess a conserved binding site on another kinetochore subunit, with the most likely candidate being the Mis12 complex subunit Dsn1, which is implicated in Csm1 recruitment in S. cerevisiae (Corbett et al. 2010; Sarkar et al. 2013). Using our ConFeaX pipeline (Tromer et al. 2016), we identified a highly conserved motif in the N-terminus of Dsn1 (Dsn1-N) that is characterised by a stretch of negatively charged residues, two conserved phenylalanine residues, and a stretch of positively charged residues (Fig. 1a, S1a). Strikingly, Dsn1 proteins in the Oomycetes appear to possess two Dsn1-N motifs, one with a canonical (negative-FF-positive) directionality and the other with an inverted (positive-FF-negative) directionality (Fig. S1b). We also identify two Dsn1-N motifs in Dsn1 orthologs of Encephalitozoon species (Fig. S1b). As budding yeast Csm1 forms a homodimer, this pattern suggests that in both Oomycetes and Encephalitozoon, a single copy of Dsn 1 may simultaneously bind both protomers of a Csm1 dimer (Fig. S1c). Overall, the phylogenetic profile of Dsn1$\mathrm{N}$ throughout eukaryotes is strikingly similar to that of Csm1 $(r=0.799)$ (Fig. 1b, c), indicative of co-evolution and supporting the idea that Dsn1-N is the kinetochore-targeting motif of Csm1 in many eukaryotic lineages. We also found that Csm1 proteins throughout eukaryotes show high conservation in the conserved hydrophobic cavity previously implicated in Dsn 1 binding (Corbett et al. 2010) (Fig. S1d).

Narrowing our analysis to fungi, we identified two major groups of species with differing conservation patterns in the Dsn1 N-terminus. Most fungi, including $S$. pombe, contain a short conserved motif matching the widely conserved Dsn1-N motif identified above (Fig. S2a, b). In contrast, in $S$. cerevisiae and other species with identified pointcentromeres and containing a Mam1 ortholog_-suggesting a 
likely role for monopolin in meiotic sister kinetochore monoorientation-Dsn1 contains the extended Box 1-2-3 region identified previously (Sarkar et al. 2013) (Fig. S2c-e). Strikingly, budding yeast Dsn1 Box 1 bears a strong resemblance to the Dsn1-N motif conserved in a wide range of eukaryotes (Fig. 2a, S2). Thus, while Dsn1-N/Box 1 appears to be an ancestral, widely conserved Csm1-targeting motif, Dsn1 Box 2-3 likely evolved as an adaptation to monopolin's role in sister kinetochore monoorientation in point-centromere fungi.

\section{Reconstitution and structure of a budding yeast Csm1-Dsn1 complex}

To better understand the interactions between the budding yeast monopolin complex and the kinetochore, and the roles of the Dsn1 Box 1, 2, and 3 regions in Csm1 binding, we sought to reconstitute a complex between Csm1 and the Dsn 1 N-terminus. We first separately purified the S. cerevisiae Csm1 globular domain (residues 69-190 of 190) and the Dsn1 Box 1-2-3 region (residues 71-110 of $576)$ and measured a binding affinity $\left(K_{d}\right)$ of $12 \mu \mathrm{M}$ by isothermal titration calorimetry (ITC) (Fig. S3a). We next coexpressed and purified a stable $S$. cerevisiae $(S c) \mathrm{Csm}^{69-}$ ${ }^{181}$ :Dsn1 $1^{71-110}$ complex (with the C-terminal 9 disordered residues of Csm1 removed), but were unable to identify crystallisation conditions for this complex. We therefore screened paralogs from several related budding yeast, and successfully purified a complex between the Candida glabrata $(C g)$ Csm1 globular domain (residues 69-181) and the Dsn1 Box 1-2-3 region (residues 14-72) (Fig. S3b, c). We identified crystallisation conditions for this complex and determined the structure to $2.3 \AA$ resolution (Table S5). The $\mathrm{Cg}$ Dsn1 Box 1-2-3 region shows high homology with the equivalent region of $S c$ Dsn1 (56\% identity and $82 \%$ similarity between $S c$ Dsn1 residues $72-110$ and $C g$ Dsn1 residues 32-67) (Fig. 2b), and we were also able to reconstitute a complex of $C g$ Csm $1^{69-181}$ (56\% identical to $S c$ Csm1 in this region) with $S c$ Dsn1 ${ }^{71-110}$ (Fig. S3d). We crystallised and determined the structure of this chimeric complex to $2.5 \AA$ resolution. We also determined a $3.0 \AA$ A-resolution structure of $C g$ Csm $1^{69-181}$ in complex with the Csm1-binding region of $C g$ Mam1 (residues 162-216) (Fig. S3e, Fig. S4). While our attempts to purify a ternary complex of Csm1, Dsn1, and Mam1 were unsuccessful, these crystal structures provide a comprehensive picture of how budding yeast Csm1 interacts through its C-terminal globular domain with Dsn1 and Mam1.

Our prior biochemical data showed that Sc Dsn1 interacts with a highly conserved hydrophobic cavity on the Csm1 globular domain (Corbett et al. 2010). Later work implicated the Dsn1 Box 1-2-3 region as necessary for binding Csm1, and mutagenesis revealed a particular requirement for the Box 2-3 region (Sarkar et al. 2013). Our structures of the $C g$
$\mathrm{Csm} 1^{69-181}: \mathrm{Dsn} 1^{14-72}$ (Fig. 2c) and $C g \mathrm{Csm} 1^{69-181}: \mathrm{SC}$ Dsn $1^{71-110}$ (Fig. 2d) complexes reveal a consistent interface between Dsn1 Box 2-3 and Csm1 (Fig. S5), while the $C g$ Csm1 ${ }^{69-181}$ :Dsn1 ${ }^{14-72}$ structure reveals a second interface between Csm1 and Dsn1 Box 1 (Fig. 2e, S6a). Therefore, all three conserved segments in the Dsn1 $\mathrm{N}$ terminus contact Csm1. Intriguingly, the conserved hydrophobic cavity on Csm1 is involved in binding both Dsn1 Box 3 (in both the $C g$ Csm1 ${ }^{69-181}:$ Dsn $^{14-72}$ and $C g$ Csm1 ${ }^{69-181}: S c$ Dsn1 $1^{71-110}$ structures) and Box 1 (in the $C g \mathrm{Csm}^{69-181}$ :Dsn1 ${ }^{14-72}$ structure), which form strikingly similar interfaces with Csm1 (Fig. $2 \mathrm{c}-\mathrm{e})$. We next sought to understand which of these interfaces are important for sister kinetochore monoorientation during meiosis.

\section{Dsn1 Box 2 contributes to successful meiosis}

In the crystal structures of both $C g C s m 1^{69-181}: D s n 1^{14-72}$ and the chimeric $C g \mathrm{Csm}^{69-181}: S c$ Dsn $1^{71-110}$ complex, the Dsn1 Box 2-3 region wraps around the Csm1 globular domain, with Box 2 forming an $\alpha$-helix that packs against the "side" of the Csm1 dimer, and Box 3 binding the Csm1 hydrophobic cavity (Fig. 2c, d; Fig. S5). Box 2 is highly conserved in yeast with point centromeres, with an alternating pattern of hydrophobic (Sc Dsn1 L88/L92/L95) and polar (Sc Dsn1 E90/N94/ D97) residues (Fig. 3a). In both structures, this region forms an $\alpha$-helix oriented with the hydrophobic residues facing outward into solution, and the polar residues packed tightly against Csm1 (Fig. 3a). This binding mode is unexpected, as hydrophobic residues are most often buried in protein-protein interfaces, rather than solvent-exposed. To determine the importance of Dsn1 Box 2 for Csm1 binding and successful meiosis, we mutated either the polar or hydrophobic residues in Box 2 and tested their function in vivo and in vitro. First, we produced the Dsn1 Box 1-2-3 region (71-110) by in vitro translation, and performed pulldown assays with purified Csm1 (Fig. 2f). This assay showed that mutation of the polar residues contacting Csm1 (Dsn1 E90, N94, and D97) to lysine did not detectably reduce Csm1 binding, while mutation to alanine appeared to increase binding (Fig. 2f). In contrast, mutation of the solvent-exposed hydrophobic residues of Dsn1 Box 2 (L88, L92, and L95) impaired binding to Csm1, with lysine substitutions having the greatest effect and alanine or aspartate substitutions causing a modest reduction in binding (Fig. 2f). This suggests that, at least when Dsn 1 Box 1 and 3 are present, mutations in the Csm1-contacting surface of Box 2 do not compromise binding, while the solventexposed hydrophobic residues play an unexpectedly important role in Csm1 binding.

To determine the role of Dsn1 Box 2 in meiosis, we generated $S$. cerevisiae strains with mutations in either the Csm1contacting polar residues (Dsn1 E90A/N94A/D97A and Dsn1 E90K/N94K/D97K) or the solvent-exposed hydrophobic 
a Dsn1-N (eukaryotes)

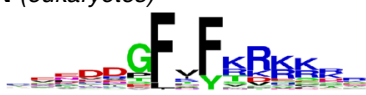

Box 1 (budding yeast)

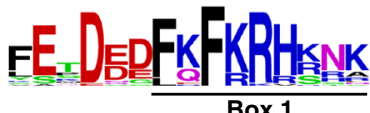

b

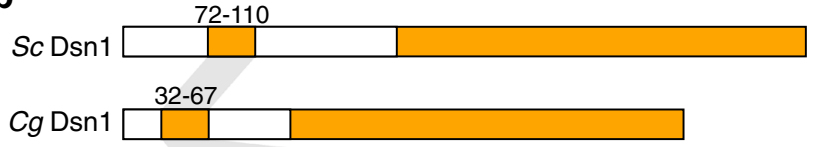

Sc Dsn1 69-113 SPDLKFKRHKNKHIQGFPTLGERLDNLQDIKKAKRVENFNSSAPI Cg Dsn1 29-70 DEGFEFRRHSN--L-GVPTLGERLDSLHEIKSARRMDHFNSSRNS Box 1
Box 2
C

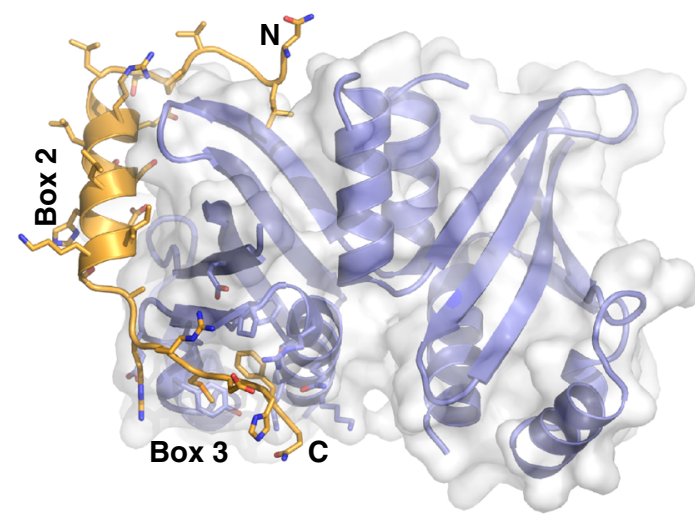

Cg Csm1 $\bigcirc g$ Dsn114-72

e

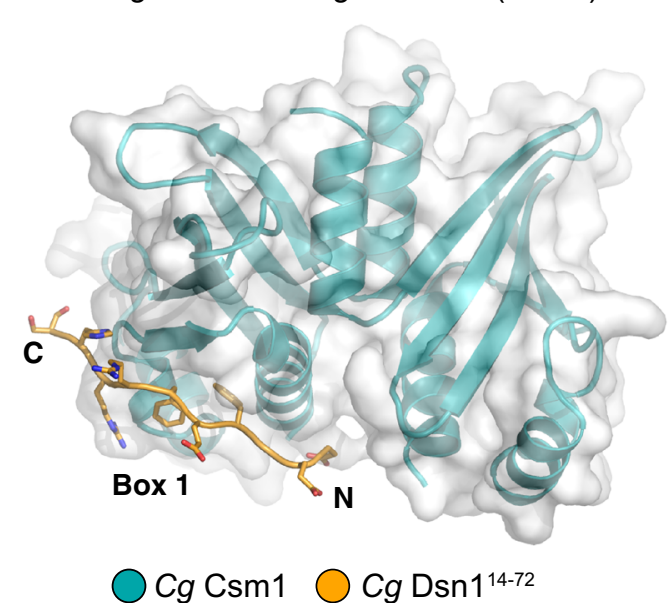

Fig. 2 Structure of the Csm1-Dsn1 complex. a Sequence logos for eukaryotic Dsn1-N (Fig. S1) and budding yeast Dsn1 Box 1 (Fig. S2a, b), demonstrating high homology between the two motifs; b Domain schematic of Dsn1 from S. cerevisiae and C. glabrata, with conserved regions shown in orange. The Dsn $1 \mathrm{C}$-terminal domain forms a folded complex with other MIND complex subunits, while the N-terminal conserved region interacts with Csm1. Bottom: Sequence alignment of the $S$. cerevisiae and C. glabrata Box 1-2-3 region (see Fig. S2 for larger sequence alignments); $\mathbf{c}$ Overall view of the $C g \mathrm{Csm}^{69-181}: C g$ Dsn $1^{14-72}$ complex, showing the Dsn1 Box 2-3 region (orange) interacting with a

residues (Dsn1 L88A/L92A/L95A). Impaired monopolin function causes mis-segregation of chromosomes during meiosis, producing aneuploid gametes which are frequently inviable. d $\quad \operatorname{Cg} \operatorname{Csm}^{69-181: S c ~ D s n 1^{11-110}}($ Box 2-3)

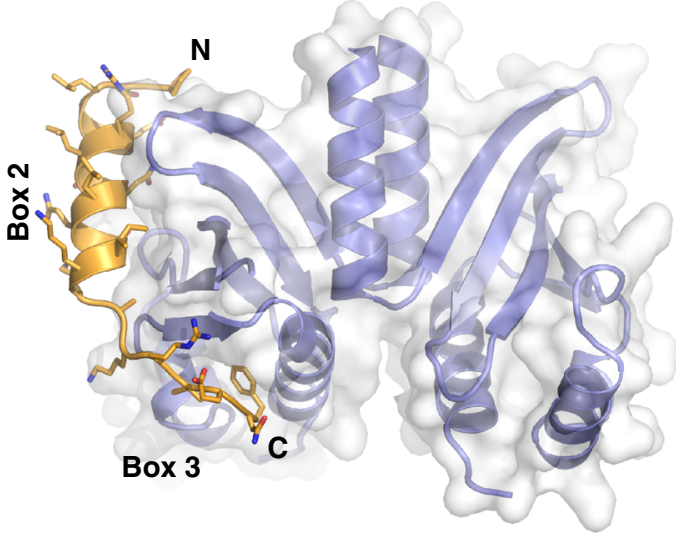

Cg Csm1 OSc Dsn171-110

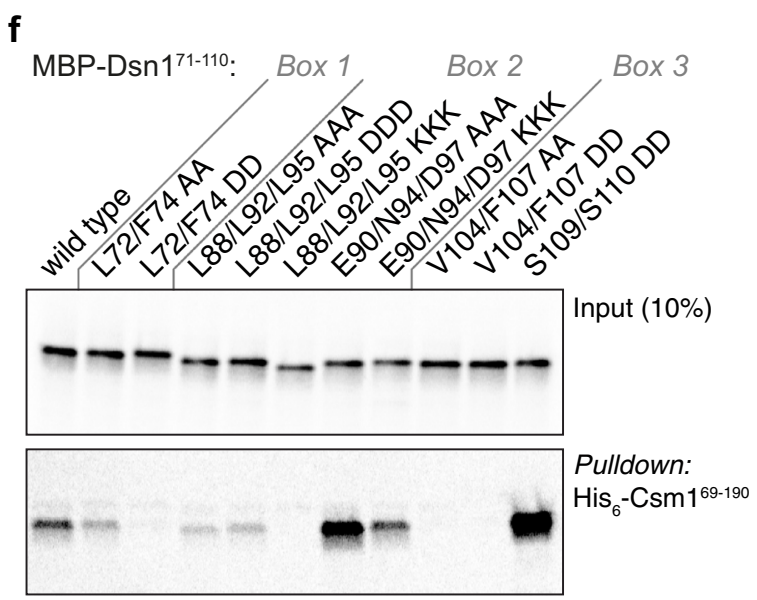

Csm1 dimer (blue with white surface); $\mathbf{d}$ Overall view of the $C g \mathrm{Csm}^{69}$ ${ }^{181}$ :Sc Dsn $1^{71-110}$ complex, showing the Dsn1 Box 2-3 region. See Fig. S5 for more details on Csm1-Dsn1 Box 2-3 interactions, and Fig. S6 for crystal packing interactions; e Overall view of the $C g$ Csm $1^{69-181}: C g$ Dsn $1^{14-72}$ complex, showing the Dsn 1 Box 1 region (orange) interacting with a Csm1 dimer (teal with white surface). See Fig. S5a for crystal packing interactions for this complex; $\mathrm{f} \mathrm{Ni}^{2+}$-pulldown of in vitro translated $S$. cerevisiae Dsn1 N-terminal region constructs by $S c$ His $_{6}-\mathrm{Csm}^{69-190}$

Therefore, we first analysed the ability of $S$. cerevisiae strains with homozygous mutations in Dsn1 to produce viable meiotic progeny, or spores. We observed that mutation of the Dsn1 


\section{a $C g$ Csm1 ${ }^{69-181}: C g$ Dsn114-72 (Box 2)}

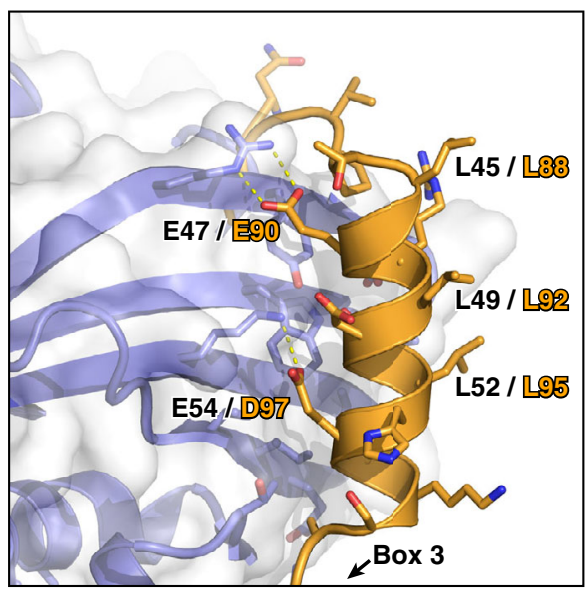

Numbering: C. glabrata / \& बerrevtlilae b Number of viable spores per meiosis

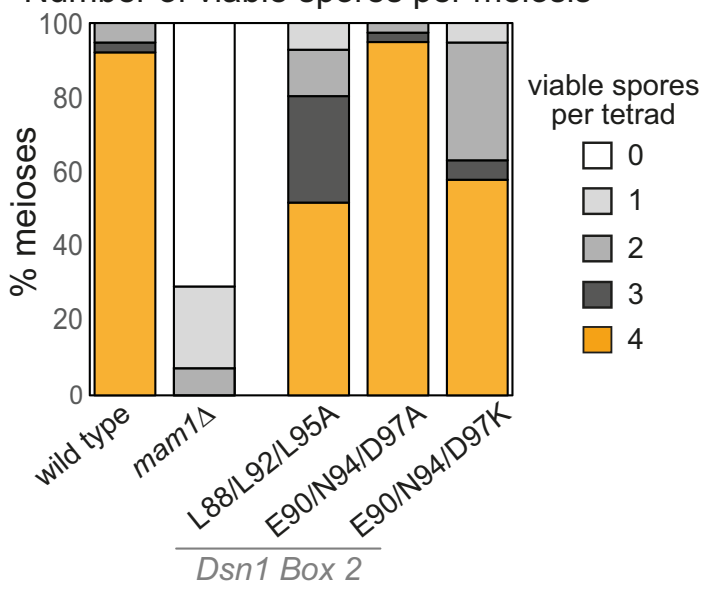

C wild type - sister kinetochore monoorientation

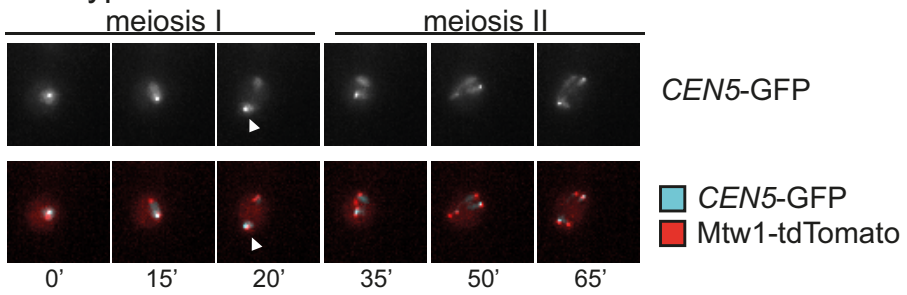

DSN1-L88/L92/L95 AAA - sister kinetochore biorientation example

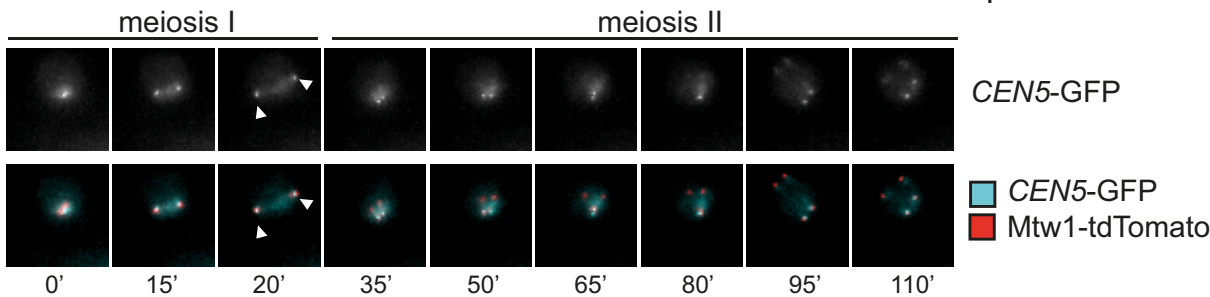

d Segregation of heterozygous

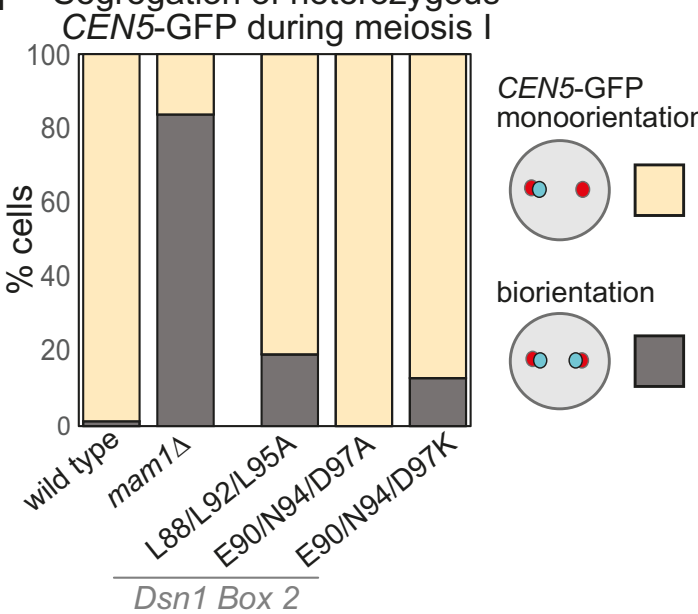

Box 2 polar residues to alanine (Dsn1-E90A/N94A/D97A) had no detectable effect on spore viability, while lysine substitutions (Dsn1-E90K/N94K/D97K) resulted in reduced spore viability (Fig. 3b), despite being proficient in Csm1 binding in vitro (Fig. 2f). Mutation of the solvent-exposed hydrophobic residues (Dsn1-L88A/L92A/L95A), which strongly affected Csm1 binding in vitro, also reduced spore viability (Fig. 3b). We next asked whether the ability to establish sister 
kinetochore monoorientation during meiosis I could underlie these effects on spore viability. We imaged live DSN1-L88A/ L92A/L95A, DSN1-E90A/N94A/D97A, and DSN1-E90K/ $N 94 K / D 97 K$ cells carrying a heterozygous CEN5-GFP marker (which tracks the segregation of a single sister chromatid pair in meiosis I; see "Materials and Methods"), a kinetochore marker (Mtw1-tdTomato), and a marker for anaphase I onset (Pds1tdTomato). Using these strains, we detected monoorientation defects consistent with each mutation's effect on spore viability: While virtually all wild type and DSN1-E90A/N94A/D97A cells segregate $C E N 5$-GFP foci to the same pole during anaphase I, segregation of CEN5-GFP foci to opposite poles was observed for $\sim 20 \%$ of DSN1-L88A/L92A/L95A and E90K/ N94K/D97K cells during anaphase I (Fig. 3c, d; Movies S1S3). We observed frequent splitting and re-association of CEN5-GFP prior to final separation of Mtw1-tdTomato into two foci which then divided into four foci (Movies S1-S3). This is characteristic of monopolin mutants where the persistence of centromere cohesion at anaphase I prevents efficient segregation of bioriented sister chromatids to opposite poles until anaphase II (Toth et al. 2000; Rabitsch et al. 2003; Petronczki et al. 2006). To confirm that the observed behaviour is due to biorientation of sister chromatids in meiosis I, we analysed metaphase I-arrested cells where spindle forces cause bioriented sister kinetochores to separate prior to anaphase I onset (Lee and Amon 2003). Consistent with our live cell

4 Fig. 3 Dsn1 Box 2 contributes to successful meiosis. a Close-up view of the $C g$ Dsn 1 Box 2 region (orange) interacting with the "side" of a Csm1 protomer (blue with white surface) in the $C g \mathrm{Csm}^{69-181}: C g$ Dsn1 ${ }^{14-72}$ complex. Residue numbers shown are for $C g$ Dsn1, with $S c$ Dsn1 equivalents shown in orange text. See Fig. S5d-f for equivalent views of the $C g \mathrm{Csm}^{69-181}: S c \mathrm{Dsn}^{71-110}$ and $C g \mathrm{Csm}^{69-181}: C g$ Dsn $1^{43-}$ ${ }^{67} \mathrm{DD}$ complexes; b Point mutations in Dsn1 affect spore survival. Diploid cells carrying the indicated homozygous mutations in DSN1 were sporulated, dissected, and the number of spores that formed colonies from each tetrad was scored. Between 38 and 56 tetrads were dissected for each condition, from a minimum of two independent diploid strains. Diploid strains used were generated from matings between AMy1827 and AMy1828 or AMy1835 (wild type), AMy1932 and AMy1947 (mam1 1 ), AMy21921 and AMy22719 (DSN1-L88A L92A L95A), AMy23151 and AMy23152 (DSN1-E90A N94A D97A), and AMy24629 and AMy24632 (DSN1-E90K N94K D97K); c, d Live cell imaging of heterozygous CEN5-GFP foci during meiosis reveals defective monoorientation in the presence of Dsn1 Box 2 mutations. Cells also carry Mtw1-tdTomato to label kinetochores and Pds1tdTomato, the destruction of which marks anaphase I onset; c Representative images of strains producing either wild type Dsn1 or Dsn1-L88A L92A L95A. While wild type cells segregate a single CEN5-GFP focus to one pole, some DSN1-L88A L92A L95A cells split GFP foci and exhibit delayed meiosis II. Arrowheads indicate position of CEN5-GFP foci during anaphase I, revealing whether they segregate to the same pole (monooriented, as in the wild type example) or opposite poles (bioriented as in DSN1-L88A L92A L95A cells). Images are from frames taken at 15 min intervals; d Scoring of GFP foci position at anaphase I onset, defined as the first occasion on which Mtw1tdTomato segregate. Strains used were AMy25832 (wild type; $n=78$ ), AMy25881 (DSN1-L88A L92A L95A; $n=26)$, AMy25763 (DSN1-E90A N94A D97A; $n=39)$ and AMy25881 (DSN1-E90K N94K D97K; $n=93)$ imaging, heterozygous CEN5-GFP foci split with increased frequency in metaphase I in DSN1-E90K/N94K/D97K cells, though we also observed a low frequency of CEN5-GFP splitting in DSN1-E90A/N94A/D97A metaphase I cells (Fig. S7). While these effects are less severe than observed in a maml $\Delta$ mutant (Fig. 3d, S7), the data nonetheless indicate that Dsn1 Box 2 is important for co-segregation of sister chromatids during anaphase I.

Although it remains possible that amino acid changes cause structural perturbations of the Dsn1 Box $2 \alpha$-helix, Box 2 mutants DSN1-L88A/L92A/L95A, DSN1-E90A/N94A/D97A or $D S N 1-E 90 K / N 94 K / D 97 K$ do not cause sensitivity to microtubule-depolymerising drugs (Fig. S8a), indicating that mitotic chromosome segregation is largely unperturbed. These findings suggest, unexpectedly, that the hydrophobic outer surface is critical for sister chromatid co-segregation during meiosis I. Interestingly, the polar residues on the Csm1-binding inner surface of the Dsn1 Box $2 \alpha$-helix can be mutated to alanine without affecting spore viability or cosegregation of sister kinetochores, at least in the presence of functional Box 1 and 3, while mutation of these residues to lysine reduces spore viability and sister chromatid cosegregation without affecting $\mathrm{Csm} 1$ binding in vitro. Potentially, the Dsn1 Box $2 \alpha$-helix is required to make additional interactions that are important for monopolin function in vivo, though we cannot rule out minor structural changes caused by these mutations.

\section{Dsn 1 Box 3 is critical for meiosis}

While Dsn1 Box 2 forms an $\alpha$-helix and associates with the "side" of the Csm1 dimer, Box 3 forms an extended conformation that packs tightly against the Csm1 conserved hydrophobic cavity (Fig. 4a; Fig. S5a-c, g, h). This binding is equivalent to the nucleolar protein Tof2, which we previously showed shares limited sequence homology with Dsn1 Box 3 (Liang et al. 2017) (Fig. S9). The core of the interaction comprises two conserved hydrophobic residues (Sc Dsn1 V104 and F107) inserted into the conserved hydrophobic cavity on Csm1. These residues are bracketed by positively charged amino acids (Sc Dsn1 K102 and R103) on the $\mathrm{N}$-terminal side, and highly conserved serine residues (Sc Dsn1 S109 and S110) on the C-terminal side (Fig. 4a; Fig. S5 g, h). Mutations in the hydrophobic residues either to alanine (DSN1-V104A/F107A) or aspartate (DSN1-V104D/F107D) abolished Csm1 binding in vitro (Fig. 2f). Consistently, Dsn1 Box 3 mutations V104A/ F107A and V104D/F107D led to a marked decrease in spore viability, whether present in single copy (heterozygous) or both copies (homozygous) (Fig. 4b). We also observed increased separation of $C E N V$-GFP labelled sister chromatids to opposite poles in anaphase 
a $\quad C g \operatorname{Csm}^{69-181}: \operatorname{Cg} \operatorname{Dsn}^{14-72}(\operatorname{Box} 3)$

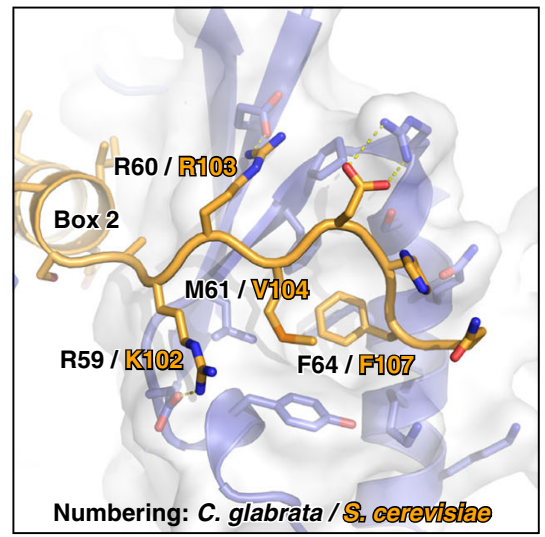

C Segregation of heterozygous

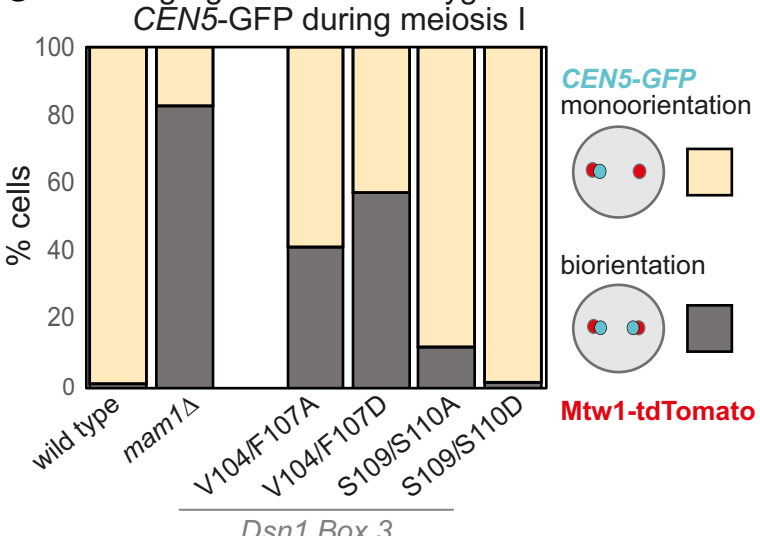

b

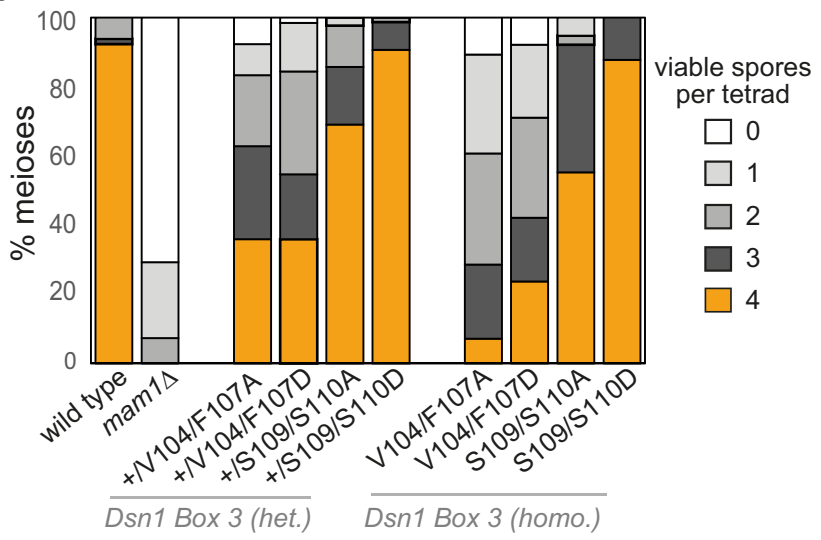

d

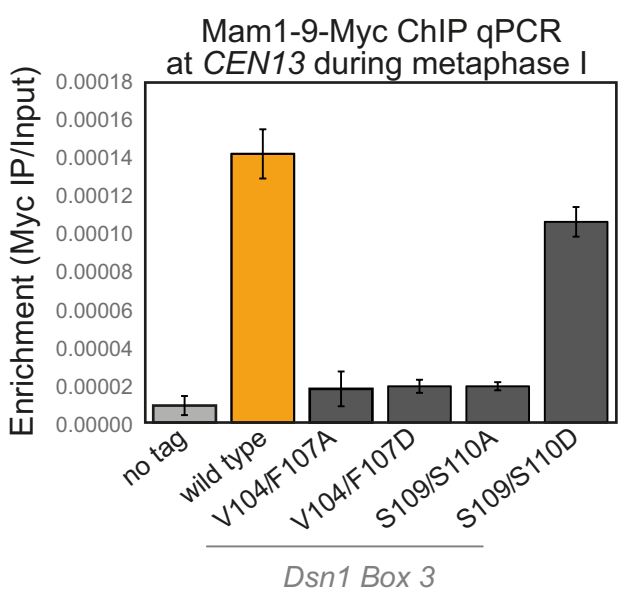

f Mam1-GFP in live cells Mam1-GFP Mtw1-tdTomato
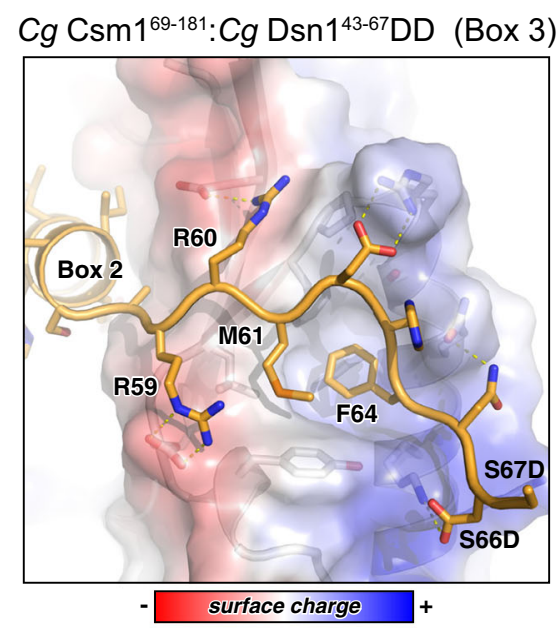
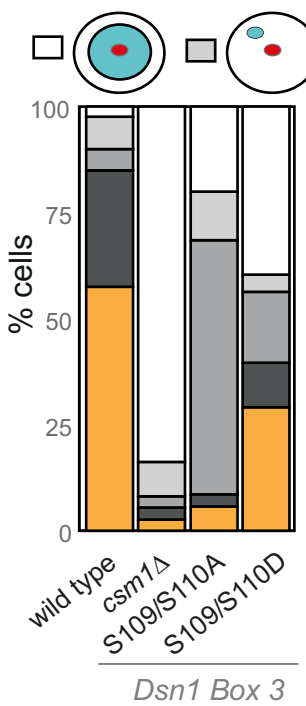

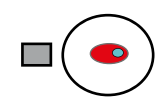

wild type
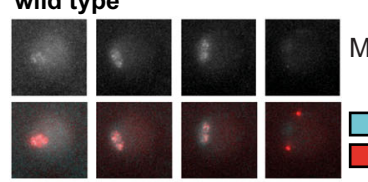

$\square$ Mam1-GFP

$\square$ Mtw1-dtTomato

$\operatorname{csm} 1 \Delta$

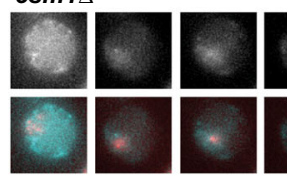

DSN1-S109/S110 AA
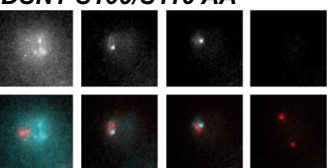

Mam1-GFP

$\square$ Mam1-GFP

$\square$ Mtw1-dtTomato
Mam1-GFP
I (Fig. 4c; Movie S4 and S5) and splitting of sister CEN5-GFP foci in metaphase I with these mutants (Fig. S7). Finally, we measured monopolin complex recruitment to kinetochores in vivo by analysing Mam1 association with a representative kinetochore by chromatin immunoprecipitation (ChIP). This assay revealed that homozygous Dsn 1 Box 3 mutations (either DSN1V104A/F107A or DSN1-V104D/F107D) caused a significant reduction in Mam1 association with kinetochores compared to wild type cells (Fig. 4d). Importantly, these effects were not due to defective kinetochore assembly, as Dsn1 Box 3 mutations did not affect overall Dsn1 
levels (Fig. S10a), and kinetochore association of both Dsn1 itself and the KMN-network protein Ndc80 was unaffected (Fig. S10b, c). Furthermore, Box 3 mutations did not result in benomyl sensitivity or cause increased chromosome loss, indicating that they did not affect mitotic chromosome segregation (Fig. S8), consistent with previous observations using Dsn 1 truncated at residue 110 (Sarkar et al. 2013). Collectively, these findings establish that the interface between Dsn1 Box 3 and Csm1 is critical for sister kinetochore cosegregation during meiosis I.

4 Fig. 4 Dsn1 Box 3 residues are critical for meiosis. a Close-up view of the $C g$ Dsn 1 Box 3 region (orange) interacting with the Csm1 conserved hydrophobic cavity (blue with white surface) in the $C g$ Csm $1^{69-181}: C g$ Dsn $1^{14-72}$ complex. Residue numbers shown are for $C g$ Dsn1, with $S c$ Dsn1 equivalents shown in orange text. See Fig. S5 $\mathrm{g}-\mathrm{i}$ for equivalent views of the $C g C s m 1^{69-181}: S c$ Dsn $1^{71-110}$ and $C g$ Csm1 $1^{69-181}: C g$ Dsn $1^{43-67}$ DD complexes; b Diploid cells with heterozygous or homozygous mutations in DSN1 were sporulated, dissected and the number of spores which grew up from each tetrad scored. Between 38 and 78 tetrads were dissected for each condition, from a minimum of two independent diploids. Data for wild type and maml $\Delta$ is reproduced from Fig. $3 \mathrm{~b}$. Heterozygous diploids were generated from crosses between AMy1827 and AMy24652 (DSN1-V104A F107A), AMy1827 and AMy25110 (DSN1-V104D F107D), AMy1827 and AMy26803 (DSN1-S109A S110A), AMy1827 and AMy24744 (DSN1-S109D S110D). Homozygous diploids were generated from crosses between AMy24624 and AMy24652 (DSN1-V104A F107A), AMy24858 and AMy25110 (DSN1-V104D F107D), AMy26426 and AMy26803 (DSN1-S109A S110A), AMy24744 and AMy24688 (DSN1-S109D $S 110 D)$; c Live cell imaging was used to score sister chromatid cosegregation during anaphase I in cells carrying heterozygous CEN5-GFP foci and Dsn1 Box 3 mutations as described in Fig. 3 c, d. Data for wild type and mam $1 \Delta$ is reproduced from Fig. $3 \mathrm{~d}$, other strains analysed and number of cells counted were AMy25762 (DSN1-V104A F107A) $n=51$, AMy26475 (DSN1-V104D F107D) $n=61$ and AMy26828 (DSN1-S109A S110A) $n=50$, AMy27009 (DSN1-S109D S110D) $n=64$; d Analysis of Mam1-9Myc association with a representative centromere $(C E N 4)$ by anti-Myc chromatin immunoprecipitation followed by quantitative PCR (ChIP-qPCR). Wild type (AM25617), DSN1-V104A F107A (AM24669), DSN1-V104D F107D (AMy26778), DSN1-S109A S110A (AMy26800) and DSN1S109D S110D (AMy26476) cells carrying MAM1-9MYC were arrested in metaphase I of meiosis by depletion of Cdc20. Strain AMy8067 was used as a no tag control. Shown is the average from 8 biological replicates for wild type and no tag. The average from 3 experiments is shown for all DSN1 mutants with the exception of DSN1-S109D S110D where the average from 5 biological replicates is shown. Error bars indicate standard error; e Close-up view of the $C g$ Dsn 1 Box 3 region with conserved serine residues mutated to aspartate (from the structure of $C g \mathrm{Csm}^{69-181}: \mathrm{Cg}$ Dsn $\left.1{ }^{43-67} \mathrm{DD}\right)$. Residue D66 is visible forming hydrogen-bond interactions with Csm1 K172. The side-chain for residue D67 is disordered, and is modelled as alanine. Csm1 is shown in white with surface coloured by charge. $\mathbf{f}$ Live cell imaging of Mam1-GFP. Cells carrying Mtw1-tdTomato were released from a prophase block by $\beta$ oestradiol-dependent inducible expression of Ndt80 (Carlile and Amon 2008). Representative images are shown for the indicated genotypes. Graph displays the fraction of cells with the localisation pattern depicted in the schematic. Strains used were wild type (AMy14942; $n=40$ ), $\operatorname{csm} 1 \Delta$ (AMy15096; $n=37$ ), DSN1-S109A S110A (AMy26963, $n=50$ ), and DSN1-S109D S110D (AMy26947, $n=39)$

\section{Phosphorylation of Dsn1 Box 3 residues may stabilise Csm1 binding}

Dsn1 Box 3 contains two serine residues (Sc Dsn1 S109 and S110) that are highly conserved throughout budding yeast (Fig. S2c, e). These serine residues are disordered in both crystal structures but are positioned close to conserved lysine residues in Csm1 ( $C g$ Csm1 K172, K175, and K179) (Fig. 4a; Fig. S5a-c, g, h). The high conservation and physical proximity of these serine residues to positively charged residues on Csm1 suggests that these residues may become phosphorylated, and that phosphorylation could reinforce the observed binding mode between Dsn1 Box 2-3 and Csm1. Indeed, mass spectrometry of Dsn1 purified from metaphase I-arrested cells showed that S109 and S110 are phosphorylated in vivo (Fig. S11). Further supporting this idea, we could reconstitute a complex of $C g \mathrm{Csm}^{69-181}$ and a minimised $C g$ Dsn 1 Box 2-3 construct (residues 43-67) with both S66 and S67 (equivalent to $S c$ Dsn1 S109 and S110) mutated to aspartate to mimic phosphorylation (referred to as $C g \mathrm{Csm} 1^{69-181}: \mathrm{Dsn} 1^{43-}$ $\left.{ }^{67} \mathrm{DD}\right)$ (Fig. S3f). We determined a $1.8 \AA$-resolution structure of this complex (Table S5), which closely agrees with both structures described above with the addition of a specific interaction between Dsn1 residue D66 and Csm1 K172 (Fig. 4e; S5b, e, h; S6c).

Consistent with the idea that phosphorylation of Dsn1 Box 3 promotes Csm1 binding, we found that Sc Dsn1 $1^{71-}$ 110 with the phosphomimetic S109D/S110D mutation showed increased binding to $S c \mathrm{Csm}^{69-190}$ in vitro (Fig. 2f). Furthermore, sister CEN5-GFP foci segregated normally to the same pole in Dsn1 S109D/S110D cells and spore viability was comparable to that of wild type cells whether one or both copies of Dsn1 carried the mutations (Fig. 4b, c; Movie S6). Consistently, ChIP and live cell imaging showed that Mam1 was localised to centromeres in cells carrying the Dsn1 S109D/S110D phosphomimetic mutations (Fig. 4d, f). We also analysed a non-phosphorylatable S109A/S110A mutant and found that, although ChIP showed that kinetochoreassociated Mam1 levels in a metaphase I arrest were reduced to a level comparable to that caused by the V104A/F107A and V104D/F104D mutations (Fig. 4d), the effect on spore viability and sister chromatid co-segregation was less pronounced (Fig. 4b, c: Movie S7) and CEN5-GFP separation at metaphase was not greatly increased (Fig. S7). Interestingly, live cell imaging of Mam1-GFP revealed a new localisation pattern in S109A/S110A cells where a single bright focus in the vicinity of kinetochores was observed (Fig. 4f). The identity of this Mam1-GFP focus remains unclear, but it could explain the ability of the S109A/S110A mutant to support sister kinetochore monoorientation. Therefore, phosphorylation of S109/S110 may be dispensable for the initial recruitment of monopolin to kinetochores, but is important for its maintenance into metaphase I. 


\section{Dsn1 Box 1 is critical for meiosis}

Sequence alignments of the Dsn $1 \mathrm{~N}$-terminal region revealed that a conserved Dsn1 Box 2-3 region is found only in those fungi with point centromeres (Meraldi et al. 2006; Westermann et al. 2007; Gordon et al. 2011) and a MAM1 gene (indicating the use of monopolin to co-orient sister chromatids during meiosis) (Ye et al. 2016) (Fig. S2c). In contrast, Dsn1 Box 1 is highly conserved throughout fungi, suggesting a possible ancestral function (Fig. 1, S2). A recent study has shown that Dsn1 Box 1 is dispensable for binding to Csm1 as measured by yeast two-hybrid assay (Sarkar et al. 2013), and our own biochemical reconstitutions reveal that the Box 2-3 region alone binds stably to Csm1, at least when the conserved Box 3 serine residues are mutated to aspartate (Fig. S3f). These findings have suggested that Dsn1 Box 1 may not be important for monopolin complex function in point-centromere fungi including $S$. cerevisiae.

Our structure of the $C g \mathrm{Csm}^{69-181}: C g$ Dsn $1^{14-72}$ complex shows how Dsn1 Box 2-3 binds Csm1, but also reveals a plausible Csm1 binding mode for Box 1. In the structure, Dsn1 Box 1 extends outward from the Csm1-Dsn1 Box 2-3 complex described above, and interacts with a crystallographic symmetry-related Csm1 monomer. This interface is remarkably similar to the Box 3 interface: Box 1 inserts two conserved hydrophobic residues ( $C g$ Dsn 1 F32 and F34, equivalent to $S c$ Dsn1 L72 and F74) into the conserved hydrophobic cavity on Csm1, and these residues are bracketed by positively and negatively charged residues that make specific interactions with Csm1 as in the Box 3 interface (Fig. 5a). Interestingly, Box 1 is oriented in the opposite direction across the Csm1 hydrophobic cavity than Box 3, with positively charged residues $(C g$ Dsn1 R35 and R36, equivalent to $S c$ Dsn1 K75 and R76) C-terminal to the hydrophobic residues, and negatively charged residues $(C g$ Dsn 1 D29 and E30, equivalent to $S c$ Dsn1 S29 and P30) N-terminal (Fig. 5a; Fig. S6). Thus, both Dsn1 Box 1 and Box 3 bind the same surface of Csm1, through similar but distinct interaction modes. This structural plasticity provides a model for how Dsn1 proteins with two Dsn1-N motifs interact with Csm1: the first motif(Dsn1-N) could bind one Csm1 protomer equivalently to Box 3, with the second (Dsn1-N2) binding a dimerrelated Csm1 protomer equivalently to Box 1. All identified Dsn1 proteins with two Dsn1-N motifs possess at least nine residues between the central hydrophobic residues in the two motifs, enough to bridge the $\sim 30 \AA$ distance between the two sites. The location of positively charged residues $\mathrm{N}$-terminal to Dsn1-N1 and C-terminal to Dsn1-N2 in these proteins (Fig. S1b) further supports this model. Importantly for monopolin's meiotic functions and unlike oomycete and Encphalitozoon Dsn1 proteins, budding yeast Dsn1 Box 1 and Box 2-3 interfaces with Csm1 are incompatible with one another, in the sense that a single Dsn1 monomer could not form both interactions with a single Csm1 dimer (Fig. S6a).
To test the importance of Dsn1 Box 1 for Csm1 binding and sister kinetochore monoorientation, we mutated Sc Dsn1 L72 and F74 to either alanine or aspartate. Both mutations reduced binding of Dsn1 to Csm1 in our in vitro pulldowns, with the L72D/F74D mutation having the greatest effect (Fig. 2f). Interestingly, these mutations also had a profound effect on spore viability, Mam1 association with centromeres during metaphase I, and segregation of sister chromatids during anaphase I (Fig. 5b-d; Movies S8 and S9), though not on benomyl sensitivity or mitotic chromosome loss (Fig. S8). Interestingly, Dsn1 Box 1 mutations caused a reduction in spore viability even when only one copy of Dsn1 carried these mutations (Fig. 5b). The presence of only a single copy of MAM1, in contrast, has no detectable effect on spore viability (Fig. $5 b)$. Truncation of the first 110 amino acids of one copy of Dsn1 was previously found to have a dominantnegative effect on meiosis (Sarkar et al. 2013). Our findings are consistent with this observation and further indicate that successful meiosis relies on the integrity of Box 1 in all copies of Dsn1. These findings strongly suggest that the interaction between Dsn 1 Box 1 and the conserved hydrophobic cavity on Csm1, as captured in the crystal structure of $C g \mathrm{Csm} 1^{69-181}: C g$ Dsn $1^{14-72}$ (Fig. 5a), is important for kinetochore monoorientation in vivo.

\section{Regional-centromere fungi utilise Dsn1 Box 1 for Csm1 binding}

Dsn1-N/Box 1 is widely conserved among eukaryotes (Fig. 1), including in S. pombe where the monopolin subunits Csm1 and Mde4 are important for mitotic chromosome segregation, but dispensable for sister monoorientation in meiosis I (Gregan et al. 2007; Choi et al. 2009) (Fig. 6a; S2a, b). The wide conservation of Dsn1-N/Box 1 suggests that it may be an ancestral monopolin-binding motif that recruits the complex to the kinetochore, potentially through an interface like that in our structure of $C g \mathrm{Csm} 1^{69-181}: C g$ Dsn $1^{14-72}$ (Fig. 6b). Supporting this idea, we have previously shown that $S$. pombe Csm1 (also called Pcs1) interacts with Dsn1 (Mis13) through its C-terminal globular domain, and that mutations to the conserved hydrophobic cavity of $S$. pombe Csm1 disrupt this interaction (Corbett et al. 2010). We used a fluorescence polarisation binding assay to test whether a peptide encoding $S p$ Dsn1-N/Box 1 (residues 5-17) is sufficient for Csm1 binding, and measured robust binding with a $K_{d}$ of $22 \mu \mathrm{M}$ (Fig. 6c). This binding was eliminated when we mutated Sp Csm1 isoleucine 241, located in the hydrophobic cavity, to aspartate (Fig. 6b). $S p$ Csm1 I241 is equivalent to $S c$ Csm1 L161, mutation of which we previously showed disrupts binding to Csm1 (Corbett et al. 2010). To further characterise this interaction, we used an in vitro pulldown assay to systematically test the importance of 


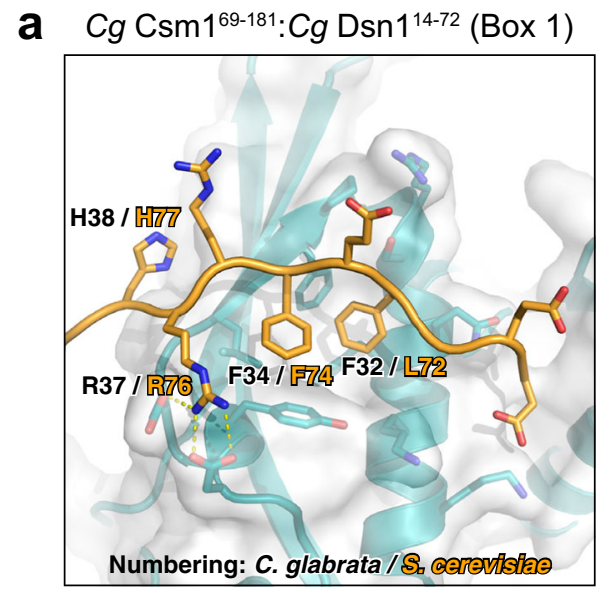

C Segregation of heterozygous CEN5-GFP during meiosis I
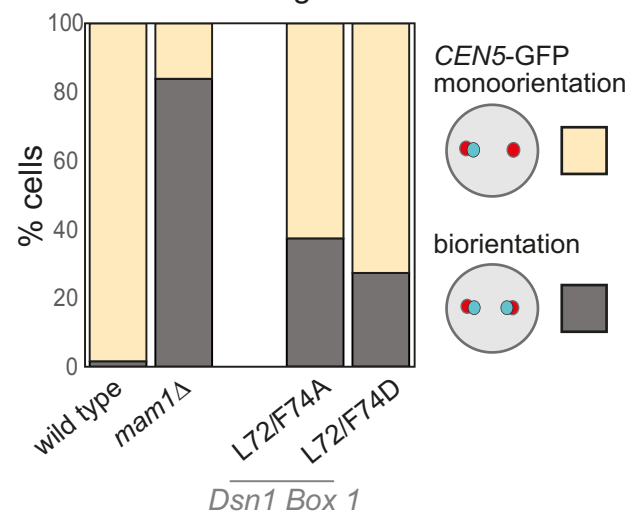

Fig. 5 The Csm1-Dsn1 Box 1 interface a Close-up view of the $C g$ Dsn 1 Box 1 region (orange) interacting with the $\mathrm{Csm} 1$ conserved hydrophobic cavity (blue with white surface) in the $C g C s m 1^{69-181}: C g$ Dsn $1^{14-72}$ complex. Residue numbers shown are for $C g$ Dsn1, with $S c$ Dsn1 equivalents shown in orange text; b Dsn1 Box 1 is critical for meiosis. Spore viability of diploid strains with the indicated genotypes were analysed as described in Fig. 3b. Between 38 and 68 tetrads were dissected for each condition, from a minimum of two independent diploids. Data for wild type and maml $\Delta$ is reproduced from Fig. $3 b$. Other diploids were generated from matings between AMy1827 and AMy11417 (heterozygous mam 1 $\Delta$ ), AMy1827 and AMy17222 (heterozygous DSN1-L72A F74A), AMy1827 and AMy17123 or AMy17313 (heterozygous DSN1-L72D L74D), AMy17222 and

conserved residues in $S p$ Dsn1-N/Box 1 (Fig. 6d). We found that Csm1 binding was disrupted upon mutation of the central hydrophobic residues (F11 and F13), upstream negatively charged residues (E6 and E9), and downstream positively charged residues (R15 and K18). These data agree closely with our structure of the $C g$ Csm1:Dsn1-N/Box 1 interface, and strongly suggest that in $S$. pombe, Dsn1N/Box 1 is primarily responsible for interactions with the Csm1:Lrs4 (Pcs1:Mde4) complex. Given the high conservation of Dsn1-N/Box 1 in all eukaryotes that possess Csm1 orthologs (Fig. 1), we propose that Dsn1-N/Box 1 comprises the ancestral, universal receptor for Csm1, while Dsn1 Box 2-3 evolved with monopolin's meiotic roles in budding yeast.
Number of viable spores per meiosis
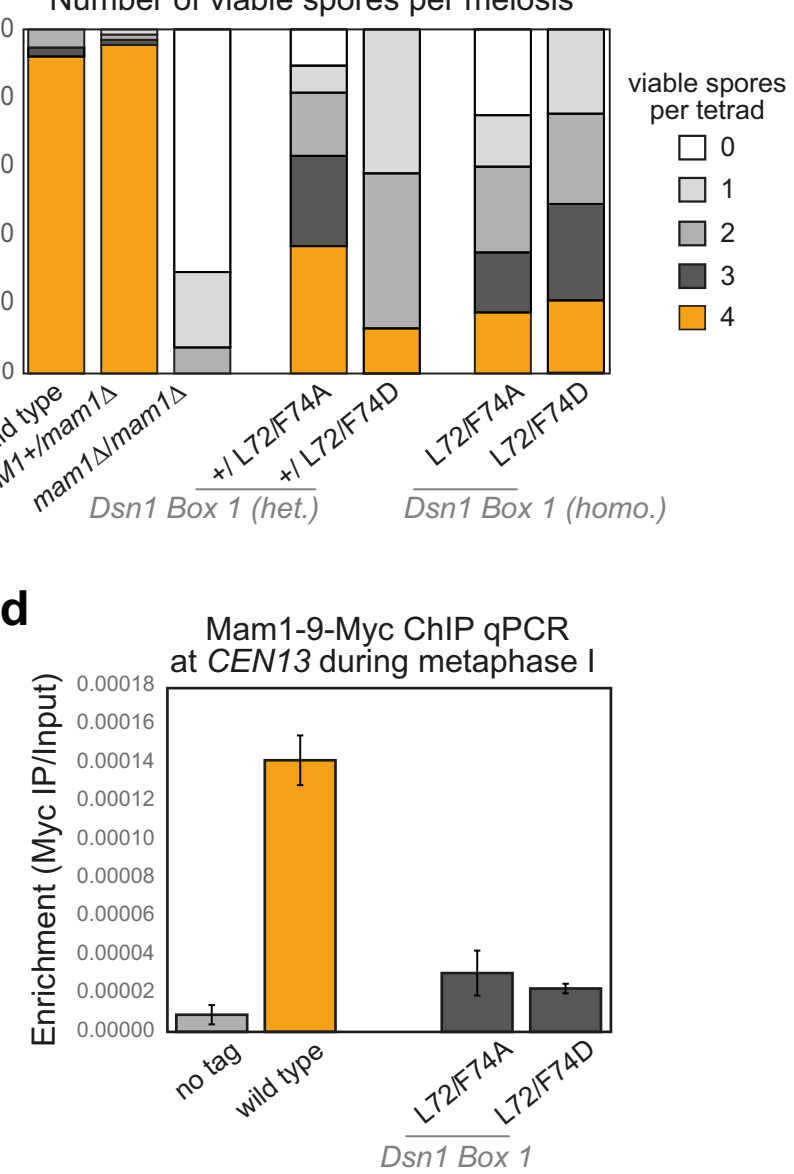

AMy17223 (homozygous DSN1-L72A F74A) or AMy17313 and AMy17373 (homozygous DSN1-L72D F72D). c Live cell imaging was used to score sister chromatid co-segregation during anaphase I in cells carrying heterozygous CEN5-GFP foci and Dsn1 Box 1 mutations as described in Fig. 3 c, d. Data for wild type and mam $1 \Delta$ is reproduced from Fig. 3 d, other strains analysed were AMy25821 (DSN1-L72A $F 74 A ; n=78)$ and AMy26543 (DSN1-L72D F72D; $n=59)$; d Mam1 association with a representative centromere in a metaphase I arrest was analysed by ChIP-qPCR as described in Fig. 4d. Data for wild type and no tag is reproduced from Fig. 4d. Other strains used were AMy25618 (DSN1-L72A F74A) and AMy26543 (DSN1-L72D F72D) and the average of 3 or 5 biological replicates, respectively, is shown with standard error bars

\section{Dsn1 Box 1 and Box 3 perform independent roles in meiosis}

We found that key residues in both Box 1 and Box 3 are important for sister kinetochore monoorientation and successful meiosis (Figs. 4, 5). While the full Dsn1 Box 1-2-3 region (residues 71-110) binds robustly to Csm1 in vitro, neither Dsn1 Box 1 (residues 71-80) or Dsn1 Box 2-3 (residues $80-110$ ) alone bound strongly in this assay (Fig. 7a). These findings suggest that robust Csm1 binding relies on the integrity of both Box 1 and Box 3. Consistently, point mutations in either Box 1 (residues L72 and F74) or Box 3 (V104 and F107) strongly reduced Csm1 binding in vitro (Fig. 2f). 
a

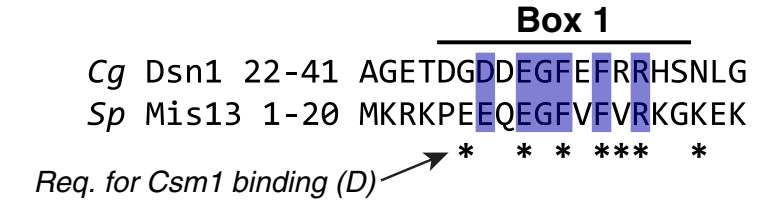

b

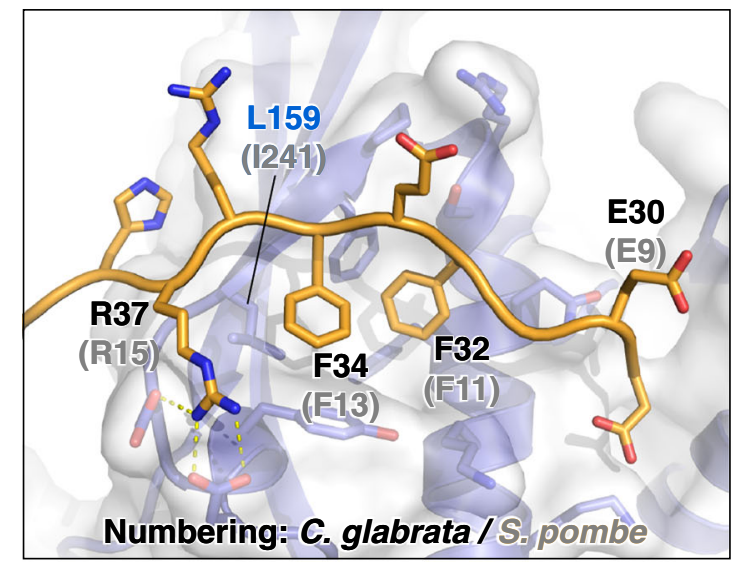

$\bigcirc$ Cg Csm1 $\bigcirc$ Cg Dsn1 29-38

Fig. 6 S. pombe Mis13 binds Csm1 through a Dsn1-N/Box 1 motif. a Sequence alignment of the $C g$ Dsn 1 Box 1 region with the N-terminal region of $S$. pombe Mis13 (See Fig. S2a-e for sequence alignment of fungal Dsn1 N-termini); b Close-up view of the $C g$ Dsn1 Box 1 region (orange) interacting with the Csm1 conserved hydrophobic cavity (blue with white surface). Residue numbers shown are for $C g$ Dsn1 (orange) and Csm1 (blue), with $S$. pombe Mis13 equivalents shown in grey text; c Fluorescence polarisation peptide-binding assay showing interaction of the isolated S. pombe Mis13 Dsn1-N region (residues 5-17, sequence

However, neither set of mutations affected spore viability as severely as deletion of MAMI or heterozygous truncation of the first 78 (removing Box 1) or 110 (removing Box 1-2-3) residues of Dsn 1 (Fig. 7b). These data raise the possibility that Dsn 1 Box 1 and Box 3 could act at least partially redundantly in vivo. To determine whether this is the case, we combined Dsn1 mutations with alanine substitutions in the hydrophobic residues of Box 1 or Box 3 and the putative phosphorylation sites S109/S110 to generate Dsn1-L72A/L74A/V104A/ F107A, Dsn1-L72A/L74A/S109A/S110A, and Dsn 1 L72A/L74A/V104A/F107A/S109A/S110A. Interestingly, spore viability after meiosis where one copy of Dsn1 carries mutations in both Box 1 and Box 3 was comparable to that of strains with Dsn $1 \mathrm{~N}$-terminal truncations $(\Delta 78$ and $\Delta 110)$, whether the $\mathrm{N}$ terminus of Dsn 1 from the other allele was intact or not (Fig. 7b). These results show that while the integrity of both Dsn1 Box 1 and Box 3 is required for robust Csm1 binding in vitro and functional monoorientation in vivo, they also have at least partially independent roles in meiosis.

\section{Discussion}

The V-shaped monopolin complex performs multiple roles in kinetochore and chromosome biology, which
C

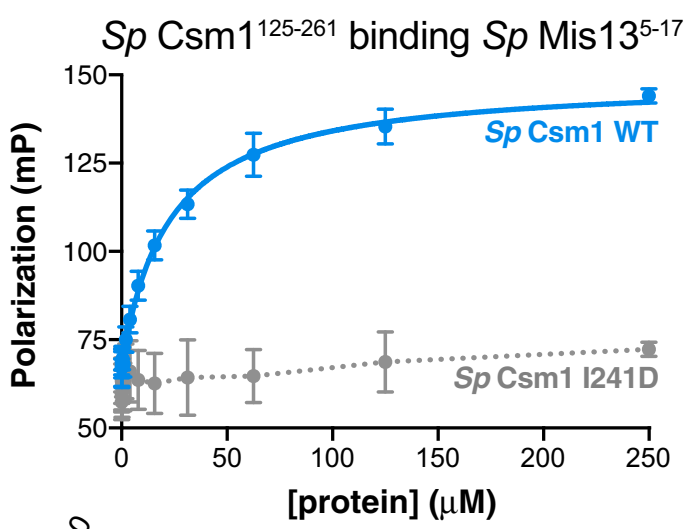

$-$<smiles>[Te][Te][Te]</smiles>

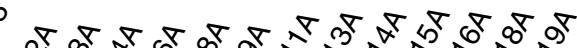

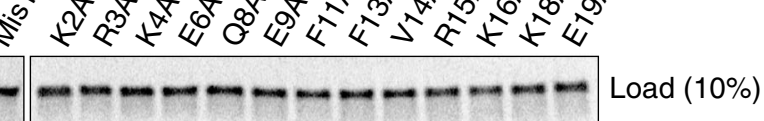
Pulldown: $\mathrm{His}_{6}-\mathrm{Sp} \mathrm{Csm} 1^{125-261}$

PEEQEGFVFVRKG) with purified S. pombe Csm1 C-terminal globular domain (residues 125-261). The Csm1 I241D mutant mimics the S. cerevisiae L161D mutant, known to disrupt binding of Dsn 1 in vitro (Corbett et al. 2010) by disrupting the conserved hydrophobic cavity (L159 in Cg Csm1; see panel B); $\mathbf{d ~ N i}^{2+}$-pulldown of in vitro translated $S$. pombe Mis 13 N-terminal region (wild type and mutants) by $\mathrm{His}_{6}$-Csm $1^{125-261}$. Mis13 residues whose mutation to alanine disrupts Csm1 binding are marked by asterisks in panel a

are likely to be achieved through its ability to act as a molecular crosslinker. This activity is mediated by binding of the two Csm1 homodimer heads, one at each apex of the $\mathrm{V}$, to short peptide motifs on target molecules to be linked together. Our previous work had identified a conserved hydrophobic cavity on the Csm1 homodimer that is important to target monopolin both to the nucleolus and to kinetochores (Corbett et al. 2010), and a second surface important for binding additional partner proteins in both contexts (Corbett and Harrison 2012; Liang et al. 2017). Here, we defined the molecular interactions between Csm1 and its kinetochore receptor, Dsn1. We unexpectedly identify two mutually exclusive modes for Dsn1-Csm1 binding, in which the same hydrophobic cavity in Csm1 can interact with distinct conserved motifs in the Dsn1 N-terminal region (Box 1 and Box 2-3, respectively). Through cell biological and biochemical studies using specific mutations (see Table 1 for summary of mutant characterisation), we demonstrate that both binding modes are important for sister kinetochore monoorientation during meiosis I in vivo. While Box 1 is the ancestral monopolin receptor at kinetochores, Box 2-3 is conserved only in yeast with point centromeres that use monopolin to direct sister kinetochore monoorientation during meiosis I. 


\section{Dsn1-N/Box 1-the ancestral monopolin recruiter}

Although Mam1 orthologs appear to be present only in budding yeast with point centromeres, we identify Csm 1 orthologs in a wide range of eukaryotes where they are likely to function at the kinetochore. In the fission yeast $S$. pombe, Csm1 and Mde4 (Lrs4) associate with kinetochores during mitosis where they prevent merotelic kinetochoremicrotubule attachments (Gregan et al. 2007). In budding yeast mitosis, Csm1 and Lrs4 associate with kinetochores in anaphase, independently of Mam1 (Brito et al. 2010). Knockout of the Arabidopsis thaliana ortholog of Csm1 (Titan-9) results in the classic titan phenotype leading to problems in endosperm development and thus suggesting a role in meiosis (Tzafrir et al. 2002; Tzafrir et al. 2004). Lastly, the highly divergent Csm1 ortholog in Dictyostelium discoideum, named Cenp-68, was found to be involved in associating clustered kinetochores to the spindle pole body-like structure during interphase (Schulz et al. 2009). Altogether, these studies indicate that Csm1 is in some way involved in bridging kinetochores during mitosis or meiosis. Strikingly however, Csm1 has been lost in most metazoans, except for the earlybranching lineages Nematostella vectensis and Amphimedon queenslandica.

Our identification of Csm1 orthologs outside fungi suggest that these organisms share a conserved monopolin interaction motif in the kinetochore. Consistent with this idea, our sequence alignments revealed that Dsn1-N/Box 1 is broadly conserved in eukaryotes and co-evolves with Csm1 (Fig. 1, $\mathrm{S} 1, \mathrm{~S} 2)$. In our structure of $C g \mathrm{Csm}^{69-181}: C g \mathrm{Dsn} 1^{14-72}$, we found that Dsn1 Box 1 forms an interaction motif that associates with the Csm1 hydrophobic cavity (Fig. 5a). Mutation of two conserved hydrophobic residues that extend into the conserved Csm1 hydrophobic cavity (L72 and F74 in $S$. cerevisiae) resulted in loss of Mam1 from kinetochores during meiosis I, a failure to properly monoorient kinetochores, and a resultant loss of spore viability (Fig. 5). This demonstrates that Dsn1 Box 1 interaction with Csm1 is critical for sister kinetochore monoorientation during meiosis I. Supporting the idea that $S$. pombe monopolin uses a similar interaction mechanism for its recruitment to kinetochores, we find that the isolated Dsn1-N/Box 1 region from $S p$ Dsn1 (called Mis13) binds Csm1 via its conserved hydrophobic cavity (Fig. 6c), and that the mutation of conserved residues in $S p$ Dsn1-N/Box 1 disrupt binding to Csm1 (Fig. 6d). Taken together, these data provide a strong argument that the Dsn1N/Box 1 represents an ancestral docking site for monopolin at kinetochores. Curiously, however, we observed no strong effects on mitotic chromosome segregation upon mutation of residues in Dsn1 Box 1 (Fig. S8b), consistent with a previous analysis of DSN1- $\Delta 110$ (Sarkar et al. 2013). Nevertheless, in S. cerevisiae, both Csm1 and Lrs4 are found at kinetochores during mitotic anaphase (Brito et al. 2010) and testing the requirement for Dsn1 Box 1 in this recruitment will be an important future priority.

\section{Dsn1 Box 2-3-an adaptation to meiotic function}

The Dsn1-N/Box 1-Csm1 interaction likely represents a general mechanism whereby monopolin can be recruited to kinetochores, but our data show that this motif on its own is insufficient for sister kinetochore monoorientation during meiosis I. Instead, both the Dsn1 Box 2-3 region and the presence of Mam1 (and by extension Hrr25) are additionally required for sister monoorientation. Structurally, Dsn1 Box 3 binds the same hydrophobic cavity on Csm1 to Dsn1 Box 1, only in the opposite orientation, with the Box $2 \alpha$-helix packing onto the side of the Csm1 homodimer. The structure of the Csm1:Dsn1 Box 3 region is also strikingly similar to that of Csm1:Tof2 (Fig. S9), which recruits Csm1 to the nucleolus to regulate ribosomal DNA silencing and recombination (Liang et al. 2017). Both Dsn1 Box 2-3 and Tof2 insert a pair of hydrophobic residues into the Csm1 hydrophobic cavity, and both possess a pair of positively charged residues immediately $\mathrm{N}$-terminal to the hydrophobic residues. Instead of two highly conserved serine residues as in Dsn1, however, Tof2 possesses a pair of conserved negatively charged residues that interact with the conserved lysine residues on Csm1 (Fig. S9). Thus, Csm1 interacts with diverse partners in the nucleolus and at kinetochores using similar mechanisms.

While a Csm1 homodimer possesses two identical conserved hydrophobic cavities capable of binding either Dsn1 Box 1 or Box 3, the orientation of the two binding modes and the proximity of Dsn1 Box 1 and Box 2-3 mean that it is impossible for a single Dsn1 protomer to simultaneously interact with Csm1 through both binding modes (Fig. S6a). Since the conserved Dsn1 Box 2-3 region is specifically found in budding yeast with point centromeres and Mam1 homologs, it is likely that Csm1 binding by Dsn1 Box 2-3 is specifically important for sister kinetochore monoorientation. Indeed, our mutational analysis of Dsn 1 Box 3 provides evidence that this is the case (Fig. 4). These observations predict the existence of meiosis I-specific mechanisms that enable Csm1-Lrs4 to engage with Dsn1 Box 2-3 and thereby fuse kinetochores. How might this be controlled? An attractive possibility is that this is the function of the Mam1-Hrr25 module of monopolin.

We previously showed that Mam1 associates with the Csm1 globular domain, and links Hrr25 to monopolin through a flexible tether (Corbett and Harrison 2012; Ye et al. 2016). In the crystal structure of Sc Csm1:Mam1, Mam1 forms an extended interface with $\mathrm{Csm} 1$ that includes an $\alpha$-helix binding to the "side" of Csm1 and a phenylalanine residue (Sc Mam1 F262) inserted into the conserved hydrophobic cavity of Csm1 (Corbett and Harrison 2012). As these data are difficult to reconcile with our finding that Dsn1 Box 2-3 interacts with the same surfaces on Csm1, we determined a new crystal 


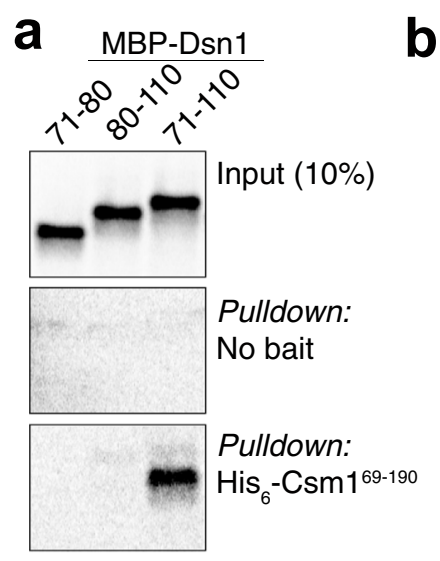

C

(1) Unstable - Box 1

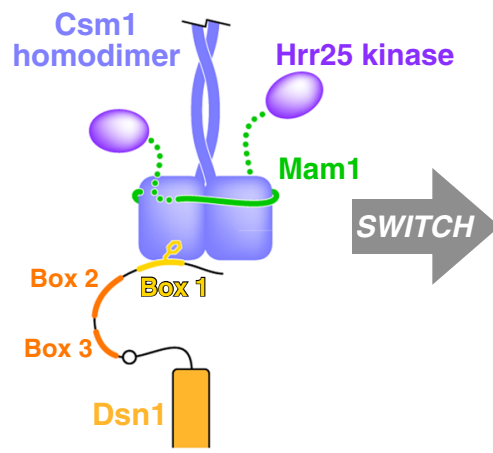

(n)
100

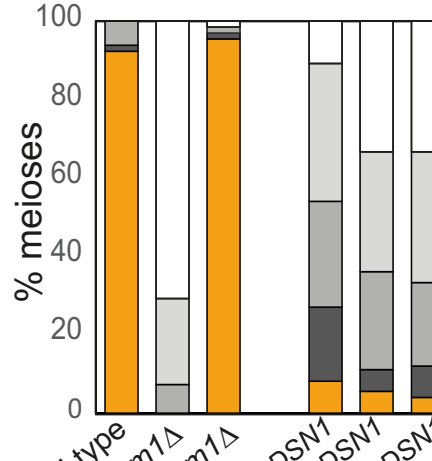

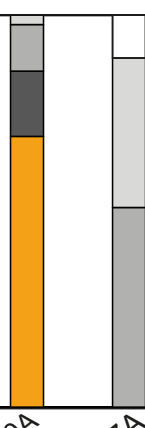
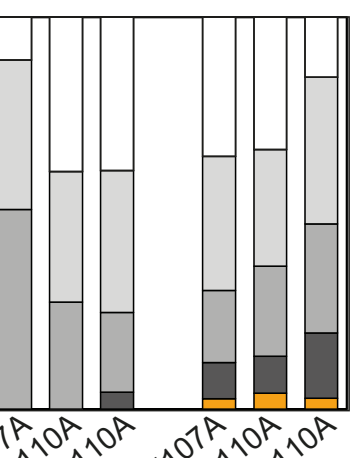

viable spores
per tetrad
$\square 0$
$\square 1$
$\square 2$
$\square 3$
$\square 4$
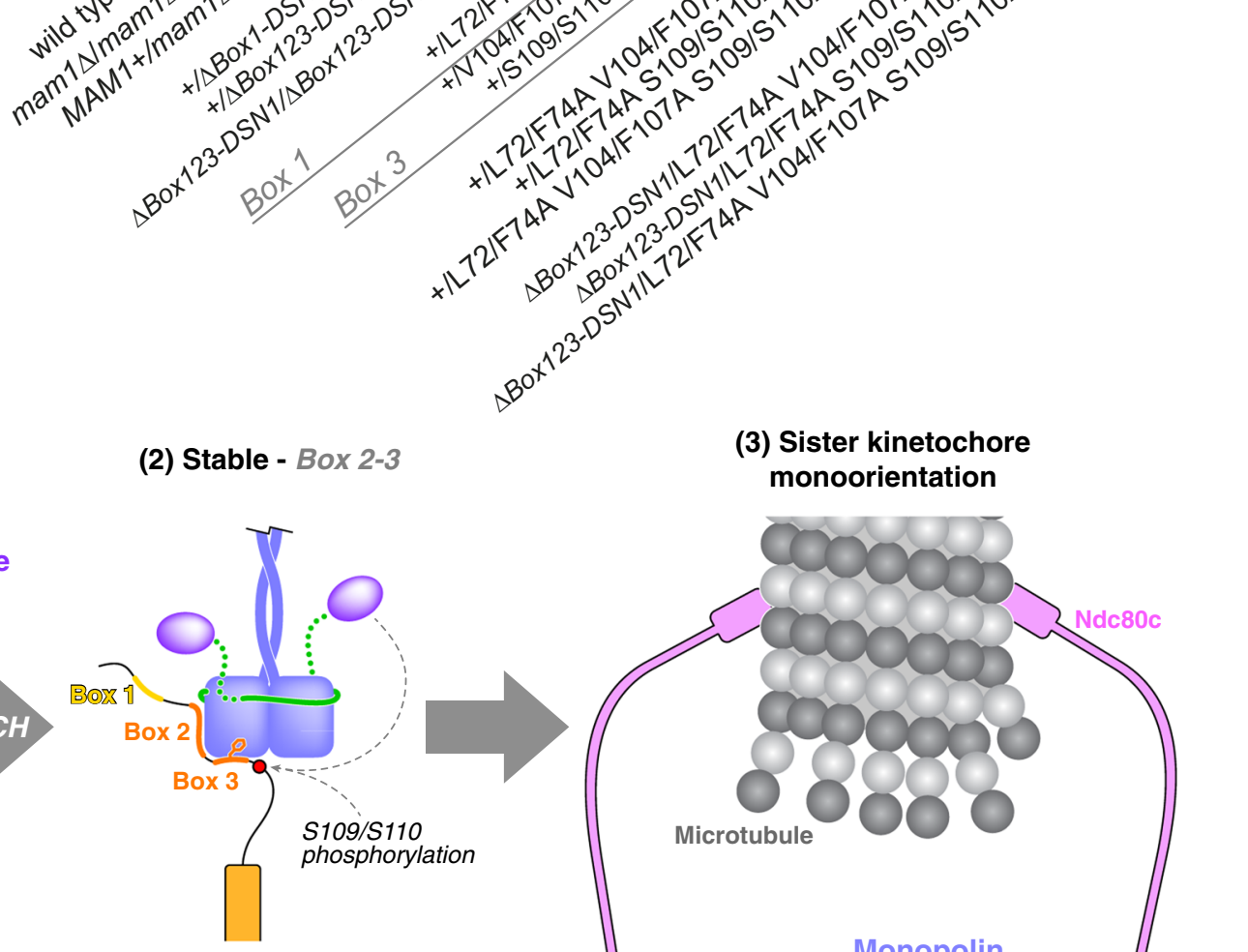

(2) Stable - Box 2-3

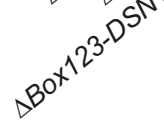

(3) Sister kinetochore monoorientation

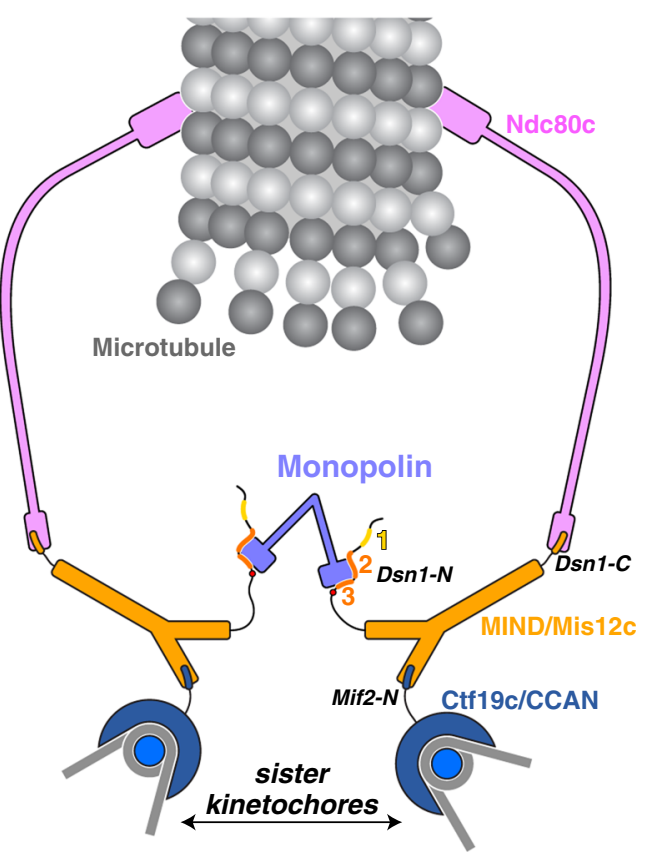

structure of a $C g$ Csm1 ${ }^{69-181}$ :Mam1 $1^{162-216}$ complex (Fig. S4). This structure closely resembles our earlier structure of the $S$. cerevisiae complex, but does not include an ordered $\alpha$ helix or any interaction with the Csm1 hydrophobic cavity. Closer inspection of sequence conservation in Mam1 orthologs reveals very poor conservation beyond the "core" region of Mam1 that interacts with Csm1 in our new structure, suggesting that the additional interactions observed in our earlier structure of the $S$. cerevisiae complex represented non-specific crystal packing interactions rather than a biologically relevant interface. Thus, our new structural data support the idea that Mam1 and Dsn1 Box 2-3 can bind simultaneously to a Csm1 protomer, rather than competing for Csm1 binding (Fig. S4d). Interestingly, our structural modelling suggests that Mam1 is positioned close to the solvent-exposed residues on the Dsn1 Box $2 \alpha$-helix (Fig. S4d), suggesting a potential further interaction that could stabilise the ternary complex and explain the role of these residues in Dsn1 Box 2 (Fig. 3). 
Taken together, our structural and functional data suggest the following general model for monopolin function at meiosis I kinetochores (Fig. 7c): The monopolin complex may first be recruited to kinetochores through an unstable interaction between Csm1 and the ancestral Box 1 motif on the Dsn1 N-terminus. Initial binding may be non-specific, with the two Csm1 homodimer "heads" interacting with Dsn1 protomers either within a single kinetochore, between sister kinetochores, or between non-sister kinetochores. We hypothesise that sister kinetochore binding by the two Csm1 heads could trigger a switch in interaction mode between Box 1 and Box 2-3, with further stabilisation of the Box 2-3 interaction coming through phosphorylation of the two conserved serine residues in Box 3 (Sc Dsn1 S109 and S110). Although the relevant kinase has yet to be identified, we note that Mam1 ideally positions Hrr25 to access Dsn1, and we have previously shown that Hrr25 phosphorylates Dsn1 in vitro, most likely in its disordered Nterminal region (Ye et al. 2016). While we did not identify specific phosphorylation sites within Dsn1, we speculate that Hrr25 may phosphorylate Dsn1 S109/S110 to stabilise monopolin-kinetochore binding. Testing the role of Hrr25, and identifying how sister kinetochore binding by the monopolin complex might be sensed, are important questions for future work.

4 Fig. 7 Dsn1 Box 1 and Box 3 perform independent roles in meiosis. a $\mathrm{Ni}^{2+}$-pulldown of in vitro translated $S$. cerevisiae Dsn1 N-terminal region constructs (fused to an $\mathrm{N}$-terminal maltose binding protein tag) by $S c$ $\mathrm{His}_{6}-\mathrm{Csm} 1^{69-190}$; b Combination of Box 1 and Box 3 mutations lead to an additive effect on meiosis. Spore viability of diploid strains with the given heterozygous mutations in DSN1 were analysed as described in Fig. 3b. Between 38 and 68 tetrads were dissected for each Dsn1 point mutant diploid, from a minimum of two independent diploids, while more than 300 tetrads were scored for the truncations. Data for wild type and mam $1 \Delta$ is reproduced from Fig. 3b. Other diploids were generated from matings between AMy17232 and AMy1827 (heterozygous $\Delta 78-D S N 1$ ), AMy17230 and AMy1827 ( $\triangle 110-D S N 1), \mathrm{AMy} 17505$ and AMy17507 (homozygous $\triangle 110-D S N 1$ ), AMy1828 and AMy26727 (wild type and DSN1-L72A F74A V104A F107A), AMy1828 and AMy25883 (wild type and DSN1-L72A F74A S109A S110A), AMy1828 and AMy26728 (wild type and L72A F74A V104A F107A S109A S110A); AMy17505 and AMy26727 ( $\triangle 110-D S N 1$ and DSN1-L72A F74A V104A F107A), AMy17505 and AMy25883 ( $\triangle 110-D S N 1$ and DSN1-L72A F74A S109A S110A), AMy17505 and AMy26728 ( $\triangle 110-D S N 1$ and $L 72 A$ F74A V104A F107A S109A S110A). Data for DSN1-L72A F74A, DSN1-V104A F107A, and DSN1-S109A S110A is reproduced from Fig. $3 b$ and $4 b$; c Model for sister kinetochore monoorientation by the monopolin complex. Initial unstable kinetochore association of the monopolin complex (1) occurs via Dsn1 Box 1 (yellow). While initial binding is non-specific, association of a single complex with sister kinetochores triggers a switch to a more stable binding mode (2) involving Dsn1 Box 2-3 (orange). The association is further stabilised by phosphorylation of Dsn1 S109/S110 by Hrr25 or another kinase, resulting in stable sister kinetochore monoorientation (3). While we draw a single Dsn1 interacting with each globular head of the monopolin complex, each head possesses two conserved hydrophobic cavities and therefore could bind two copies of Dsn1
An interesting feature of mutations in the Dsn1 Box 1-2-3 region is that they impair sister kinetochore monoorientation in a dominant fashion when heterozygous, despite the presence of an estimated 6-7 copies of Dsn1 at each kinetochore (Joglekar et al. 2006; Dimitrova et al. 2016). One explanation for this finding is that robust sister monoorientation relies on every copy of Dsn1 being able to associate with Csm1 through both identified binding modes, perhaps because each individual Csm1-Dsn1 interaction is relatively weak. A second possibility is that the level of Hrr25 activity at kinetochores is important, and reduced binding of monopolin in heterozygous DSN1 mutant strains leads to a reduction in Hrr25 activity, and a consequent reduction in the efficiency of sister kinetochore monoorientation and successful meiosis.

\section{Monopolin-a general-purpose molecular crosslinker}

While the detailed molecular mechanisms underlying specific sister kinetochore crosslinking by monopolin remain unknown, the data we present here essentially complete the structural picture of how the monopolin complex is assembled and how it interacts with kinetochores in meiosis I. When considered alongside our extensive prior structural analysis of the monopolin complex and its interactions with various binding partners, a picture emerges of a complex that is strikingly flexible in its ability to scaffold important architectural and signalling complexes in $S$. cerevisiae. The V-shaped Csm1-Lrs4 complex has two Csm1 homodimer heads positioned $\sim 10 \mathrm{~nm}$ apart, and each Csm1 homodimer head possesses four protein-protein interaction surfaces: two conserved hydrophobic cavities, and two binding sites for Mam1/Ulp2like molecules. In the nucleolus, Csm1 binds Tof 2 via the conserved hydrophobic cavity, in the process likely crosslinking multiple copies of the repetitive rDNA to suppress illegitimate recombination, and aiding Sir2-dependent transcriptional silencing (Huang et al. 2006; Mekhail et al. 2008; Corbett et al. 2010; Liang et al. 2017). At the same time, Csm1 binds Ulp2 which deSUMOylates and stabilises other key rDNA-silencing proteins (Liang et al. 2017). At the kinetochore, Csm1 binds both Dsn1 Box 1 and Box 3 via the hydrophobic cavity to directly crosslink sister kinetochores, and also binds Mam1 to indirectly recruit Hrr25. Finally, we have recently identified another Csm1 binding partner, Dse3, whose biological functions are not known but that binds Csm1 in a Ulp2/Mam1-like manner (Singh and Corbett 2018). This constellation of monopolin-interacting proteins may not yet be complete, with the Saccharomyces Genome Database (http:// yeastgenome.org) listing 70 proteins as identified physical interactors of Csm1 alone (Singh and Corbett 2018). Thus, the monopolin complex represents a remarkably versatile molecular crosslinker that has been recruited into at least three, and potentially more, functional roles in budding yeast. 


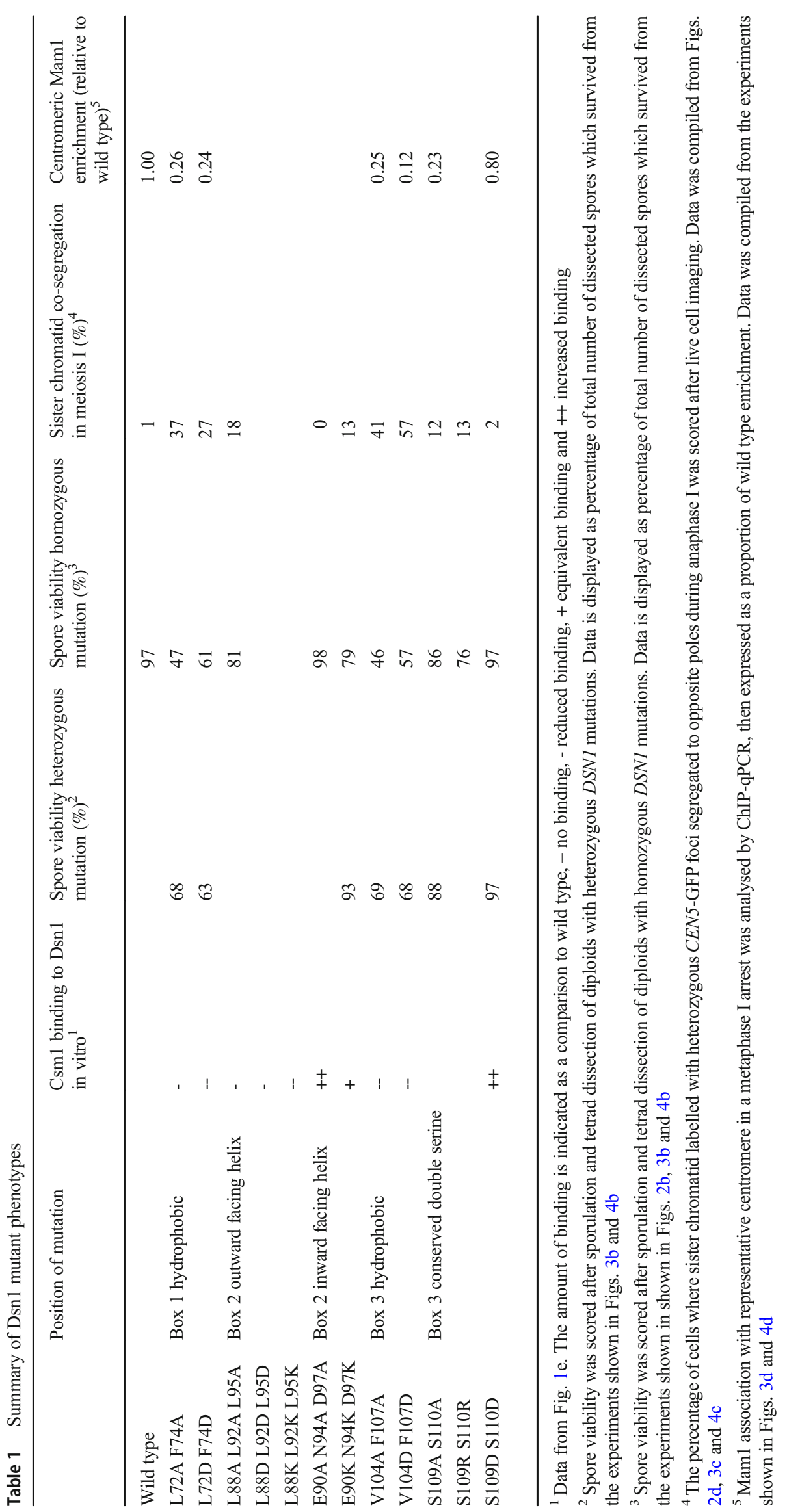


Acknowledgements We are grateful to Weronika Borek for comments on the manuscript, Dave Kelly for help with imaging, staff at the Stanford Synchrotron Radiation Lightsource and Argonne National Laboratory NE-CAT beamlines for assistance with crystallographic data collection, and Andrey Bobkov (Sanford Burnham Prebys Medical Discovery Institute) for assistance with isothermal titration calorimetry.

Funding information This work was supported by Wellcome through Senior Research Fellowships to AM [107827] and JR [103139], core funding for the Wellcome Centre for Cell Biology [203149] and a Sir Henry Wellcome Fellowship to ED [096078]. KDC acknowledges past support from the Ludwig Institute for Cancer Research and current support from the National Institutes of Health [R01 GM104141]. RP gratefully acknowledges a studentship from the Medical Research Council. ET is supported by a postdoctoral fellowship from the Herchel Smith Fund, Cambridge, UK. Work in the lab of GJPLK is supported by Oncode Institute, which is partly funded by the Dutch Cancer Society. BS is financially supported by the Netherlands Organization for Scientific Research (NWO) grant 016.160.638 (Vici).

Open Access This article is distributed under the terms of the Creative Commons Attribution 4.0 International License (http:// creativecommons.org/licenses/by/4.0/), which permits unrestricted use, distribution, and reproduction in any medium, provided you give appropriate credit to the original author(s) and the source, provide a link to the Creative Commons license, and indicate if changes were made.

\section{References}

Afonine P V, Grosse-Kunstleve RW, Echols N, et al (2012) Towards automated crystallographic structure refinement with phenix.refine. Acta Crystallogr Sect D, Biol Crystallogr 68:352-367. https://doi. org/10.1107/S0907444912001308

Akiyoshi B, Nelson CR, Ranish JA, Biggins S (2009) Quantitative proteomic analysis of purified yeast kinetochores identifies a PP1 regulatory subunit. Genes Dev 23:2887-2899. https://doi.org/10.1101/ gad.1865909

Bailey TL, Boden M, Buske FA, Frith M, Grant CE, Clementi L, Ren J, Li WW, Noble WS (2009) MEME SUITE: tools for motif discovery and searching. Nucleic Acids Res 37:W202-W208. https://doi.org/ 10.1093/nar/gkp335

Benjamin KR, Zhang C, Shokat KM, Herskowitz I (2003) Control of landmark events in meiosis by the CDK Cdc28 and the meiosisspecific kinase Ime2. Genes Dev 17:1524-1539. https://oi.org/10. $1101 / \operatorname{gad} .1101503$

Brito IL, Monje-Casas F, Amon A (2010) The Lrs4-Csm1 monopolin complex associates with kinetochores during anaphase and is required for accurate chromosome segregation. Cell cycle (Georgetown, Tex) 9: 3611-3618. https://doi.org/10.4161/cc.9.17.12885

Burge C, Karlin S (1997) Prediction of complete gene structures in human genomic DNA. J Mol Biol 268:78-94. https://doi.org/10.1006/ jmbi.1997.0951

Burrack LS, Applen Clancey SE, Chacón JM, Gardner MK, Berman J (2013) Monopolin recruits condensin to organize centromere DNA and repetitive DNA sequences. Mol Biol Cell 24:2807-2819. https://doi.org/10.1091/mbc.E13-05-0229

Carlile TM, Amon A (2008) Meiosis I is established through divisionspecific translational control of a cyclin. Cell 133:280-291. https:// doi.org/10.1016/j.cell.2008.02.032

Choi SH, Péli-Gulli M-P, Mcleod I, Sarkeshik A, Yates JR III, Simanis V, McCollum D (2009) Phosphorylation state defines discrete roles for monopolin in chromosome attachment and spindle elongation. Curr Biol 19:985-995. https://doi.org/10.1016/j.cub.2009.05.042

Clyne RK, Katis VL, Jessop L, Benjamin KR, Herskowitz I, Lichten M, Nasmyth K (2003) Polo-like kinase Cdc5 promotes chiasmata formation and cosegregation of sister centromeres at meiosis I. Nat Cell Biol 5:480-485. https://doi.org/10.1038/ncb977

Corbett KD, Harrison SC (2012) Molecular architecture of the yeast monopolin complex. Cell Rep 1:583-589. https://doi.org/10.1016/ j.celrep.2012.05.012

Corbett KD, Yip CK, Ee L-S, Walz T, Amon A, Harrison SC (2010) The monopolin complex crosslinks kinetochore components to regulate chromosome-microtubule attachments. Cell 142:556-567. https:// doi.org/10.1016/j.cell.2010.07.017

Cox J, Mann M (2008) MaxQuant enables high peptide identification rates, individualized p.p.b.-range mass accuracies and proteomewide protein quantification. Nat Biotechnol 26:1367-1372. https:// doi.org/10.1038/nbt.1511

Cox J, Neuhauser N, Michalski A et al (2011) Andromeda: a peptide search engine integrated into the MaxQuant environment. J Proteome Res 10:1794-1805. https://doi.org/10.1021/pr101065j

Crooks GE, Hon G, Chandonia J-M, Brenner SE (2004) WebLogo: a sequence logo generator. Genome Res 14:1188-1190. https://doi. org/10.1101/gr.849004

Delorenzi M, Speed T (2002) An HMM model for coiled-coil domains and a comparison with PSSM-based predictions. Bioinformatics 18:617-625

Dimitrova YN, Jenni S, Valverde R, Khin Y, Harrison SC (2016) Structure of the MIND complex defines a regulatory focus for yeast kinetochore assembly. Cell 167:1014-1027.e12. https://doi.org/10. 1016/j.cell.2016.10.011

Dosztányi Z, Csizmók V, Tompa P, Simon I (2005) IUPred: web server for the prediction of intrinsically unstructured regions of proteins based on estimated energy content. Bioinformatics 21:3433-3434. https://doi.org/10.1093/bioinformatics/bti541

Duro E, Marston AL (2015) From equator to pole: splitting chromosomes in mitosis and meiosis. Genes Dev 29:109-122. https://doi.org/10. $1101 /$ gad.255554.114

Eddy SR (2011) Accelerated profile HMM searches. PLoS Comput Biol 7:e1002195. https://doi.org/10.1371/journal.pcbi.1002195

Emsley P, Lohkamp B, Scott WG, Cowtan K (2010) Features and development of Coot. Acta Crystallogr Sect D, Biol Crystallogr 66:486501. https://doi.org/10.1107/S0907444910007493

Evans PR, Murshudov GN (2013) How good are my data and what is the resolution? Acta Crystallogr Sect D, Biol Crystallogr 69:1204 1214. https://doi.org/10.1107/S0907444913000061

Fernius J, Nerusheva OO, Galander S, Alves FL, Rappsilber J, Marston AL (2013) Cohesin-dependent association of scc $2 / 4$ with the centromere initiates pericentromeric cohesion establishment. Curr Biol 23:599-606. https://doi.org/10.1016/j.cub.2013.02.022

Gordon JL, Byrne KP, Wolfe KH (2011) Mechanisms of chromosome number evolution in yeast. PLoS Genet 7:e1002190. https://doi.org/ 10.1371/journal.pgen.1002190

Gregan J, Riedel CG, Pidoux AL, Katou Y, Rumpf C, Schleiffer A, Kearsey SE, Shirahige K, Allshire RC, Nasmyth K (2007) The kinetochore proteins Pcs1 and Mde4 and heterochromatin are required to prevent merotelic orientation. Curr Biol 17:1190-1200. https://doi.org/10.1016/j.cub.2007.06.044

Hieter P, Mann C, Snyder M, Davis RW (1985) Mitotic stability of yeast chromosomes: a colony color assay that measures nondisjunction and chromosome loss. Cell 40:381-392

Huang J, Brito IL, Villén J et al (2006) Inhibition of homologous recombination by a cohesin-associated clamp complex recruited to the rDNA recombination enhancer. Genes Dev 20:2887-2901. https:// doi.org/10.1101/gad.1472706

Joglekar AP, Bouck DC, Molk JN, Bloom KS, Salmon ED (2006) Molecular architecture of a kinetochore-microtubule attachment site. Nat Cell Biol 8:581-585 
Jurrus E, Engel D, Star K, Monson K, Brandi J, Felberg LE, Brookes DH, Wilson L, Chen J, Liles K, Chun M, Li P, Gohara DW, Dolinsky T, Konecny R, Koes DR, Nielsen JE, Head-Gordon T, Geng W, Krasny R, Wei GW, Holst MJ, McCammon JA, Baker NA (2018) Improvements to the APBS biomolecular solvation software suite. Protein Sci 27:112-128. https://doi.org/10.1002/pro.3280

Kabsch W (2010) XDS. Acta Crystallogr Sect D, Biol Crystallogr 66: 125-132. https://doi.org/10.1107/S0907444909047337

Katoh K, Standley DM (2013) MAFFT multiple sequence alignment software version 7: improvements in performance and usability. Mol Biol Evol 30:772-780. https://doi.org/10.1093/molbev/mst010

Koshland D, Hieter P (1987) [22] visual assay for chromosome ploidy. Methods Enzymol 155:351-372. https://doi.org/10.1016/00766879(87)55024-8

Lee BH, Amon A (2003) Role of polo-like kinase CDC5 in programming meiosis I chromosome segregation. Science 300:482-486

Liang J, Singh N, Carlson CR, Albuquerque CP, Corbett KD, Zhou H (2017) Recruitment of a SUMO isopeptidase to rDNA stabilizes silencing complexes by opposing SUMO targeted ubiquitin ligase activity. Genes Dev 31:802-815. https://doi.org/10.1101/gad.296145.117

Maaten LVD, Hinton G (2008) Visualizing Data using t-SNE. J Mach Learn Res 9:2579-2605

Matos J, Lipp JJ, Bogdanova A, Guillot S, Okaz E, Junqueira M, Shevchenko A, Zachariae W (2008) Dbf4-dependent CDC7 kinase links DNA replication to the segregation of homologous chromosomes in meiosis I. Cell 135:662-678. https://doi.org/10.1016/j.cell.2008.10.026

McCoy AJ, Grosse-Kunstleve RW, Adams PD, et al (2007) Phaser crystallographic software. J Appl Crystallogr 40:658-674. https://doi. org/10.1107/S0021889807021206

Mekhail K, Seebacher J, Gygi SP, Moazed D (2008) Role for perinuclear chromosome tethering in maintenance of genome stability. Nature 456:667-670. https://doi.org/10.1038/nature07460

Meraldi P, McAinsh AD, Rheinbay E, Sorger PK (2006) Phylogenetic and structural analysis of centromeric DNA and kinetochore proteins. Genome Biol 7:R23. https://doi.org/10.1186/gb-2006-7-3-r23

Olsen JV, Macek B, Lange O, Makarov A, Horning S, Mann M (2007) Higher-energy C-trap dissociation for peptide modification analysis. Nat Methods 4:709-712. https://doi.org/10.1038/nmeth1060

Otwinowski Z, Minor W (1997) Processing of X-ray diffraction data collected in oscillation mode. pp 307-326

Petronczki M, Matos J, Mori S, Gregan J, Bogdanova A, Schwickart M, Mechtler K, Shirahige K, Zachariae W, Nasmyth K (2006) Monopolar attachment of sister kinetochores at meiosis I requires casein kinase 1. Cell 126:1049-1064. https://doi.org/10.1016/j.cell.2006.07.029

Rabitsch KP, Petronczki M, Javerzat J-P, Genier S, Chwalla B, Schleiffer A, Tanaka TU, Nasmyth K (2003) Kinetochore recruitment of two nucleolar proteins is required for homolog segregation in meiosis I. Dev Cell 4:535-548

Rappsilber J, Ishihama Y, Mann M (2003) Stop and go extraction tips for matrix-assisted laser desorption/ionization, nanoelectrospray, and LC/MS sample pretreatment in proteomics. Anal Chem 75:663-670

Rumpf C, Cipak L, Schleiffer A, Pidoux A, Mechtler K, TolićNørrelykke IM, Gregan J (2010) Laser microsurgery provides evidence for merotelic kinetochore attachments in fission yeast cells lacking Pcs1 or Clr4. Cell cycle (Georgetown, Tex) 9:3997-4004. https://doi.org/10.4161/cc.9.19.13233

Sarangapani KK, Duro E, Deng Y, Alves FL, Ye Q, Opoku KN, Ceto S, Rappsilber J, Corbett KD, Biggins S, Marston AL, Asbury CL (2014) Sister kinetochores are mechanically fused during meiosis I in yeast. Science 346:248-251. https://doi.org/10.1126/science.1256729

Sarkar S, Shenoy RT, Dalgaard JZ, Newnham L, Hoffmann E, Millar JBA, Arumugam P (2013) Monopolin subunit Csml associates with MIND complex to establish monopolar attachment of sister kinetochores at meiosis I. PLoS Genet 9:e1003610. https://doi.org/10. 1371/journal.pgen. 1003610
Schulz I, Erle A, Gräf R, Krüger A, Lohmeier H, Putzler S, Samereier M, Weidenthaler S (2009) Identification and cell cycle-dependent localization of nine novel, genuine centrosomal components in Dictyostelium discoideum. Cell Motil Cytoskeleton 66:915-928. https://doi.org/10.1002/cm.20384

Shevchenko A, Wilm M, Vorm O, Mann M (1996) Mass spectrometric sequencing of proteins silver-stained polyacrylamide gels. Anal Chem 68:850-858

Singh N, Corbett KD (2018) The budding-yeast RWD protein Csm1 scaffolds diverse protein complexes through a conserved structural mechanism. Protein Sci 27:2094-2100. https://doi.org/10.1002/pro.3515

Stanke M, Tzvetkova A, Morgenstern B (2006) AUGUSTUS at EGASP: using EST, protein and genomic alignments for improved gene prediction in the human genome. Genome Biol 7:S11. https://doi.org/ 10.1186/gb-2006-7-s1-s11

Tada K, Susumu H, Sakuno T, Watanabe Y (2011) Condensin association with histone H2A shapes mitotic chromosomes. Nature 474:477483. https://doi.org/10.1038/nature10179

Tropea JE, Cherry S, Waugh DS (2009) Expression and purification of soluble His(6)-tagged TEV protease. Methods Mol Biol (Clifton, NJ) 498:297-307. https://doi.org/10.1007/978-1-59745-196-3_19

Toth A, Rabitsch KP, Galova M et al (2000) Functional genomics identifies monopolin: a kinetochore protein required for segregation of homologs during meiosis. Cell 103:1155-1168

Tromer E, Bade D, Snel B, Kops GJPL (2016) Phylogenomics-guided discovery of a novel conserved cassette of short linear motifs in BubR1 essential for the spindle checkpoint. Open Biol 6. https:// doi.org/10.1098/rsob.160315

Tzafrir I, McElver JA, Liu Cm C-M et al (2002) Diversity of TITAN functions in Arabidopsis seed development. Plant Physiol 128:38-51

Tzafrir I, Pena-Muralla R, Dickerman A, Berg M, Rogers R, Hutchens S, Sweeney TC, McElver J, Aux G, Patton D, Meinke D (2004) Identification of genes required for embryo development in Arabidopsis. Plant Physiol 135:1206-1220. https://doi.org/10. 1104/pp.104.045179

van Hooff JJ, Tromer E, van Wijk LM et al (2017a) Evolutionary dynamics of the kinetochore network in eukaryotes as revealed by comparative genomics. EMBO Rep 18:1559-1571. https://doi.org/10. 15252/embr.201744102

van Hooff JJE, Snel B, Kops GJPL (2017b) Unique phylogenetic distributions of the Ska and Daml complexes support functional analogy and suggest multiple parallel displacements of Ska by Dam1. Genome Biol Evol 9:1295-1303. https://doi.org/10.1093/gbe/evx088

Vincenten N, Kuhl L-M, Lam I, Oke A, Kerr ARW, Hochwagen A, Fung J, Keeney S, Vader G, Marston AL (2015) The kinetochore prevents centromere-proximal crossover recombination during meiosis. Elife 4:923. https://doi.org/10.7554/eLife.10850

Westermann S, Drubin DG, Barnes G (2007) Structures and functions of yeast kinetochore complexes. Annu Rev Biochem 76:563-591. https://doi.org/10.1146/annurev.biochem.76.052705.160607

Winn MD, Ballard CC, Cowtan KD, et al (2011) Overview of the CCP4 suite and current developments. Acta Crystallogr Sect D, Biol Crystallogr 67: 235-242. https://doi.org/10.1107/S0907444910045749

Winey M, Morgan GP, Straight PD, Giddings TH Jr, Mastronarde DN (2005) Three-dimensional ultrastructure of Saccharomyces cerevisiae meiotic spindles. Mol Biol Cell 16:1178-1188

Wu J, Kasif S, DeLisi C (2003) Identification of functional links between genes using phylogenetic profiles. Bioinformatics 19:1524-1530

Ye Q, Ur SN, Su TY, Corbett KD (2016) Structure of the Saccharomyces cerevisiae Hrr25:Mam1 monopolin subcomplex reveals a novel kinase regulator. EMBO J 35:2139-2151. https://doi.org/10.15252/ embj.201694082

Publisher's note Springer Nature remains neutral with regard to jurisdictional claims in published maps and institutional affiliations. 